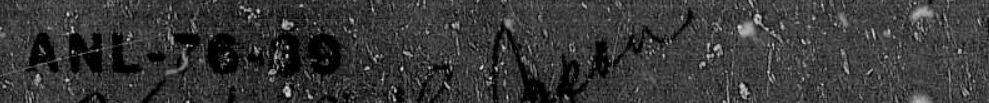

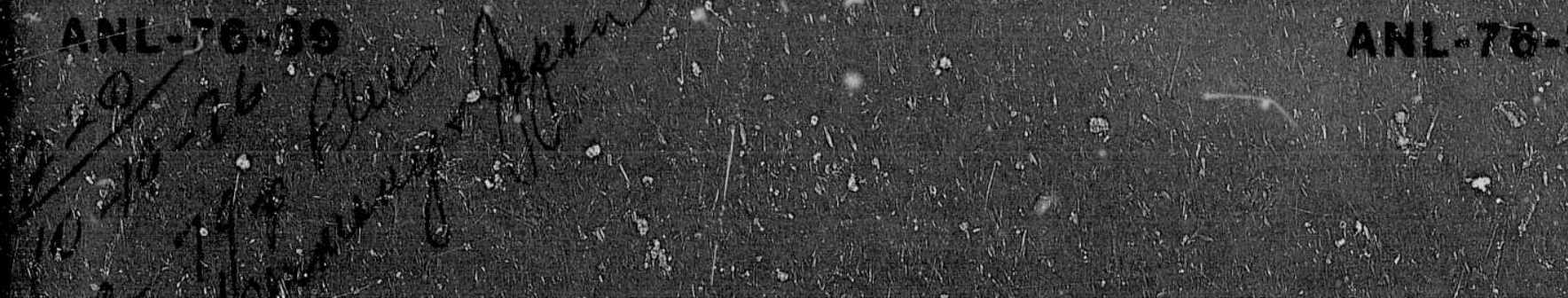

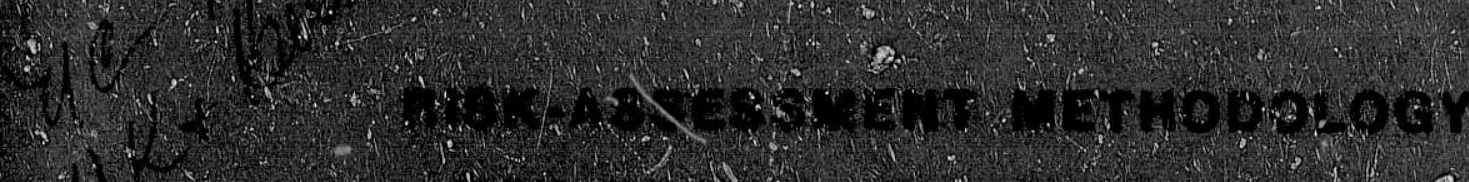

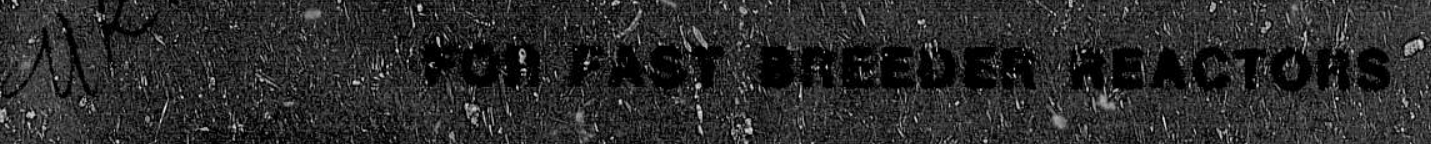
her:

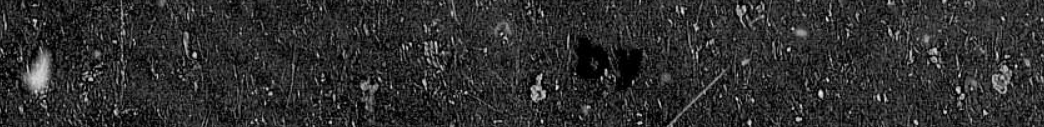

4.

(5)

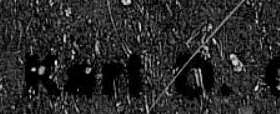

(1)

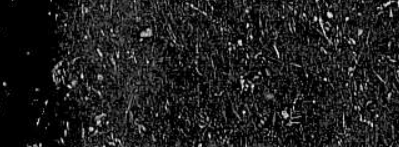

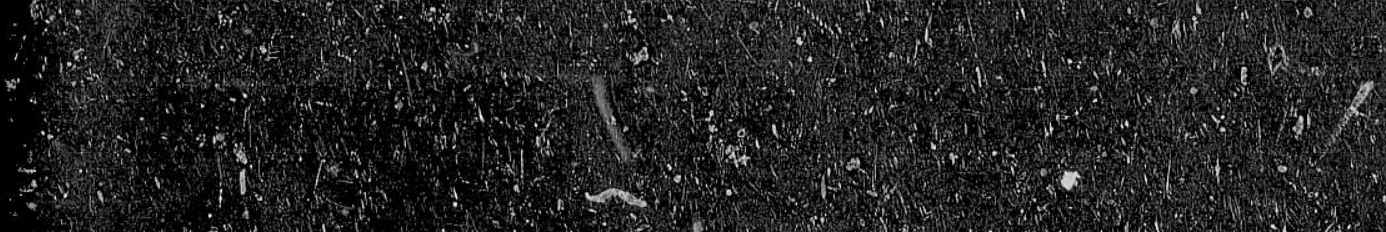

and

3

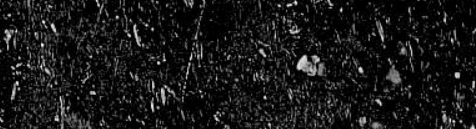

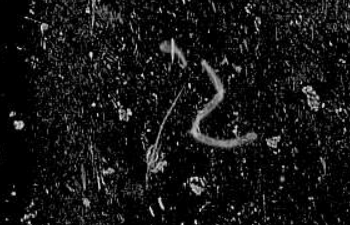

rit.
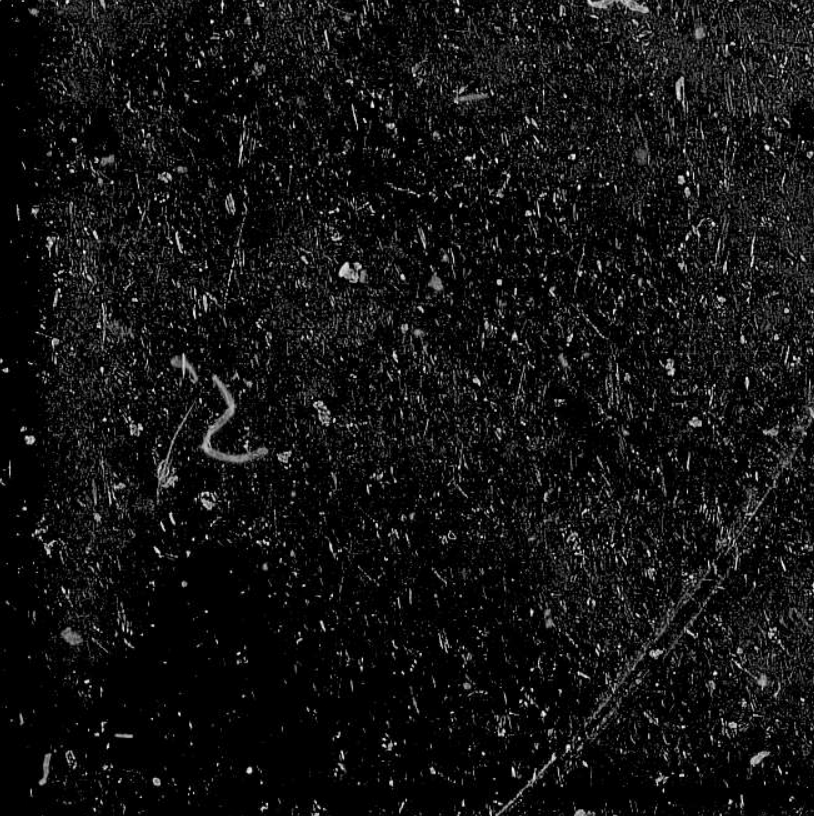

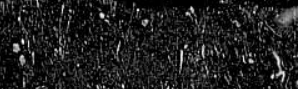

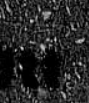

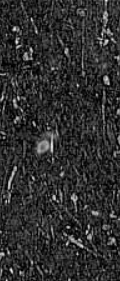

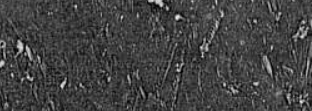

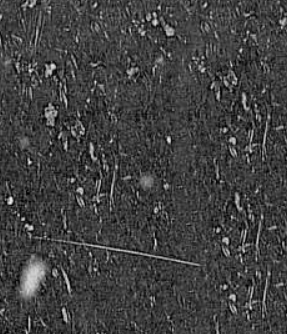




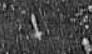




\section{ARGONNE NATIONAL LABORATORY 9700 South Cass Avenue Argonne, Illinois 60439}

\section{RISK-ASSESSMENT METHODOLOGY FOR FAST BREEDER REACTORS*}

by

Karl O. Ott

Purdue University

April 1976
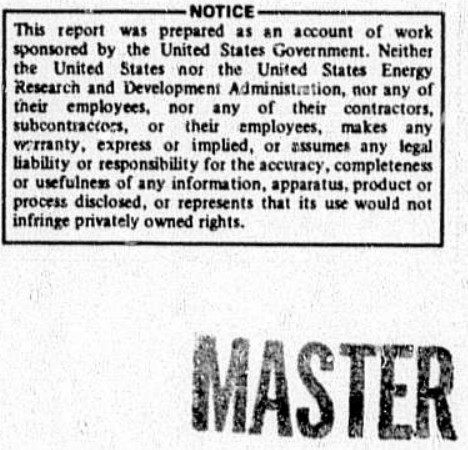

* The major part of this work was performed at Argonne National Laboratory, Reactor Analysis and Safety Division, during a leave of absence from Purdue University as recipient of the 1973 AUA-A.NL Distinguished Appointment Award 

TABLE OF CONTENTS

$\underline{\text { Page }}$

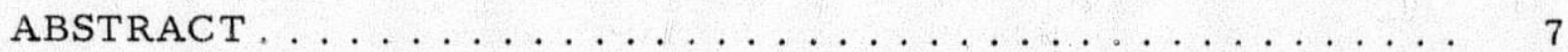

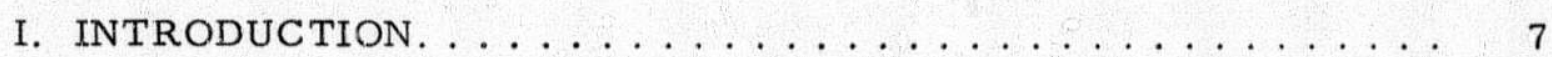

II. SURVEY OF SAFETY AND RISK-ASSESSMENT METHODS . . . . 11

A. Consequence-oriented Approaches .............. 11

1. The Maximum-credible-accident (MCA) Approach ..... 11

2. The Design-basis-accident (DBA) Approach. . . . . . . . 12

B. Concepts of Risk-oriented (Probabilistic) Approaches . . . . . 13

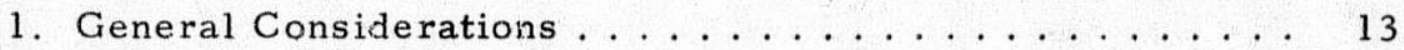

2. Farmer's Limit-line Approach . . . . . . . . . . . 14

3. Accident-risk Envelopes .............................. 15

4. Probability Distributions of Consequences. . . . . . . . 16

5. The Rasmussen Approach. . . . . . . . . . . . 17

6. Limit-line and Envelope Integrations .......... 18

C. Description of the Cause-Cons equence Relation . . . . . . . 20

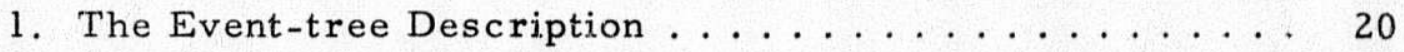

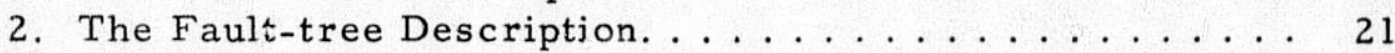

3. Accident-progression Diagrams .............. 23

4. Simulation Languages and Computer Models ... . . . . 24

D. The Accident-spectra-progression Approach . . . . . . . 25

1. The Principal Impact of Uncertainties ... . . . . . 25

2. Brief Outline of Accident-spectra-progression Approach . . 26

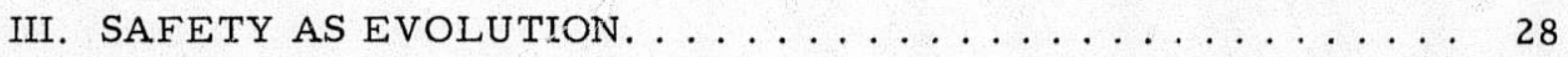

A. The Multiphase Character of Developments .......... 28

B. Long-term Testing of Safety Assessment. . . . . . . . . 29

C. Risks in Perspective ................. 30

IV. A GEINERALIZED RISK-ASSESSMENT METHODOLOGY FOR

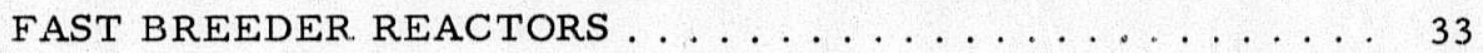

A. Introduction, Objective, and Goals of the Generalized

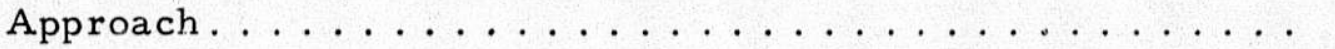


TABLE OF CONTENTS

$\underline{\text { Page }}$

B. The Accident-spectra-progression (ASP) Approach . ...... 33

1. Accident-progression Schematics ............ 33

2. Basic Concepts of the ASP Approach . . . . . . . . . 37

3. Description and Formulation of the ASP Approach ...... 41

4. Discussion of Significance and Feasibility of the ASP Approach . . . . . . . . . . . . . . 48

C. General Comparison with Other Methods . . . . . . . . 50

1. Comparison with an Analysis Comprising Deterministic Scenarios ........................ 50

2. Comparison with Farmer's Limit-line Approach ...... 52

3. Comparison with Envelope Integration ........... 53

4. Comparison with the Rasmuss en Approach . . . . . . . . 54

5. Summary and Conclusions ................. 55

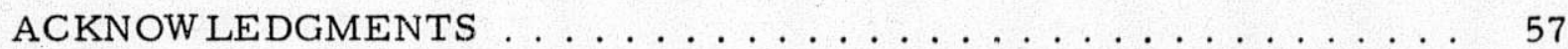

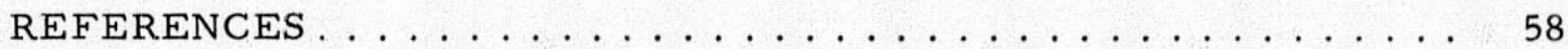




\section{LIST OF FIGURES}

No.

Title

$\underline{\text { Page }}$

1. Farmer's Limit Line or Release Criterion. . . . . . . . . . . 14

2. Illustration of a $\underline{R} i s k$ Envelope . . . . . . . . . . . 15

3. Release Frequency vs Release Categories . . . . . . . . . . . 17

4. Simplified Event Trees for Large LOCA . . . . . . . . . 22

5. Example of an Accident-progression Diagram. . . . . . . 23

6. Benefit-Risk Pattern for Involuntary Exposure . . . . . . . . 31

7. Illustration of an Accident-progression Schematic . . . . . . . 35

8. Illustration of a Consequence Distribution $\mathrm{p}_{\mathrm{C}}(\mathrm{C})$ and a Failure

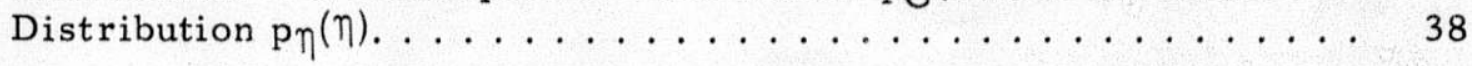

9. Illustration of the Construction of a Parameter Distribution ... . 49

10. Qualitative Comparison of Results of the ASP and of the Deterministic Scenario Approaches. . . . . . . . . . . 51

11. Comparis on of Results of the ASP and Farmer's Limit-line

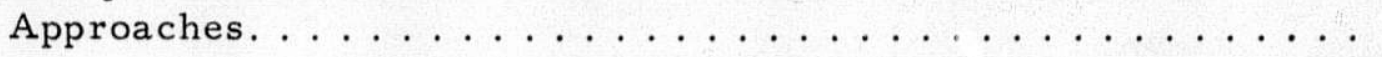

12. Comparison of Results of the ASP and the Envelope-integration Approaches. . . . . . . . . . . . . . . . .

TABLE

No.

$\underline{\text { Title }}$

$\underline{\text { Page }}$

I. Individual Risk of Acute Fatality by Various Causes. . . . . . . 30 
NOMENCLATURE

\begin{tabular}{|c|c|c|c|}
\hline a (general) & parameter, subject to uncertainty & $n$ & upper j value for parameters in caused failure calculation \\
\hline$a_{1}$ & synonym with $x_{i}=$ parametric variation of initial conditions & p (general) & nf cobability distribution \\
\hline$a_{2}, \ldots, a_{m}$ & parameters in consequence calculation & $\mathrm{Pa}(\mathrm{a})$ & protability distribution of parameter ${ }^{\circ} \mathrm{a}$ \\
\hline$a_{m+1}, \ldots, a_{n}$ & parameters in calculation of caused failures & $P_{a j}\left(a_{j}\right)$ & probability distribution of parameter ${ }_{{ }_{j}}$ \\
\hline $\mathrm{a}(\mathrm{C})$ & inverse function of $\mathrm{C}(\mathrm{a})$ & $P_{C}(C)$ & $\begin{array}{l}\text { probability distribution of consequences; consequence } \\
\text { distribution }\end{array}$ \\
\hline acc & $\begin{array}{l}\text { superscript denoting all "incidents" } \\
\text { superscript denoting ail "incidents" that progress into an } \\
\text { accident (used only temporarily; normally deleted) }\end{array}$ & $\left.{ }^{P} C^{(C ;} ; a_{1}, \ldots, a_{m}\right)$ & $\begin{array}{l}\text { consequence distribution for set of parameters } a_{1}, \ldots, a_{m} \text {. } \\
\text { i.e., parametric consequence distribution }\end{array}$ \\
\hline ASP & Accident Spectra Progression (approach) & $P_{C}^{k}\left(C_{k}\right)$ & $P_{C}(C)$ at end of Phase $k$ \\
\hline C (general) & $\begin{array}{l}\text { index denoting branches in the accident-progression schematics } \\
\text { accident consequence (intermediate or final) }\end{array}$ & $\begin{array}{l}P_{C}^{k}\left(C_{k} ; a_{i}, \ldots . a_{m}\right) \\
P_{C}^{i k b}\left(C_{k}\right)\end{array}$ & $\begin{array}{l}\text { parametric consequence distribution at end of Phase } k \\
\text { consequence distribution at end of Phase } k \text { in branch o for } \\
\text { initiator category } i\end{array}$ \\
\hline C(a) & consequence calculated for parameter a & $P_{C}^{k b}\left(C_{k}\right)$ & $p_{C}^{i k b}\left(C_{k}\right)$ for all categories i \\
\hline $\begin{array}{l}\left.\mathrm{Cla}_{1}, \ldots, \mathrm{a}_{\mathrm{m}}\right) \\
\mathrm{c}^{\mathrm{i}}\end{array}$ & $\begin{array}{l}\text { consequence calculated for set of parameters } a_{l}, \ldots, a_{\text {fn }} \\
\text { consequence for a specific accident scenario obtained through } \\
\text { deterministic calculation for initiator } i\end{array}$ & $P_{x_{i}}\left(x_{i}\right)$ & $\begin{array}{l}\text { short for a consequence distribution for initiator category i } \\
\text { after a phase identified in the text (e.g., Phase } 10) \\
\text { protability distribution of variable initiators in category i }\end{array}$ \\
\hline $\bar{c}^{i}$ & $\begin{array}{l}\text { average consequence for initiator i, calculated from } \\
\text { consequence distribution }\end{array}$ & $P_{\eta}(\eta)$ & probability distribution of caused failure of degree $\eta$ \\
\hline$C(\eta)$ & inverse function of $\eta(\mathrm{C})$ & $\begin{array}{l}\left.P_{\eta}^{(\eta ;} ; a_{1}, \ldots, a_{n}\right) \\
P_{\eta}^{(\eta \mid C)}\end{array}$ & $\begin{array}{l}\text { parametric probability distribution for caused failure } \eta \\
\text { conditiona! probability for } \eta \text { under the condition C }\end{array}$ \\
\hline CDA & core-disruptive accident & $P_{\eta}\left(\eta \mid C ; a_{m+1}, \ldots, a_{n}\right)$ & $\begin{array}{l}\text { parametric conditional probability distribution for caused } \\
\text { failure } \eta\end{array}$ \\
\hline$C R$ & containment response to pressure or melt-through & $P$ (general) & probahility (dimensionless quantity) \\
\hline f (general) & $\begin{array}{l}\text { frequency distribution; dimension: events per year and per } \\
\text { event magnitude unit }\end{array}$ & $p_{\mathrm{kb}}^{i}$ & $\begin{array}{l}\text { probability for an accident of initiating category i to pass } \\
\text { Phases } 1 \text { through } k \text { along branch b }\end{array}$ \\
\hline$f_{k}^{i}(C)$ & $\begin{array}{l}\text { frequency distribution of consequences, i.e., consequences per } \\
\text { year and } C \text { unit } \\
f(C) \text { for initiator category } i \text { at the end of accident phase } k\end{array}$ & $\begin{array}{l}p_{k}^{i} \\
p_{t r}^{i}(k, b)\end{array}$ & $\begin{array}{l}p_{k}^{i} \text { summed over all branches } \\
\text { probability for transfer just through Phase } k \text { (i.e., from } \\
k-1 \text { through } k \text { ) along branch } b\end{array}$ \\
\hline$f_{k}(C)$ & $f_{k}^{i}(C)$ for all initiator categories & $p_{\|}^{i}$ & $\begin{array}{l}\text { probability for successful termination of accident of initiating } \\
\text { category } i\end{array}$ \\
\hline $\begin{array}{l}f_{x_{i}}\left(x_{i}\right) \\
f^{d e t}\end{array}$ & $\begin{array}{l}\text { frequency distribution of the continuously varying initiator } x_{i} \\
\text { frequency distribution analogue for deterministic treatment of } \\
\text { accident scenarios, i.e., without uncertainty distributions in } \\
\text { parameters }\end{array}$ & $\begin{array}{l}P_{O C} \\
P_{C}\end{array}$ & $\begin{array}{l}\text { probability for zero consequences } \\
\text { probability for nonzero consequences } \\
\text { risk rate }\end{array}$ \\
\hline $\mathrm{F}$ (general) & $\begin{array}{l}\text { frequency in events per year. Note: } F \text { may be larger than } 1 \text {, } \\
\text { e.g., if } F \text { describes pin failure. In some publications, } F \text { is } \\
\text { denoted by } P \text { and is called "probability." }\end{array}$ & $\begin{array}{l}R \text { (general) } \\
\bar{R}\end{array}$ & $\begin{array}{l}\text { risk rate } \\
\text { average risk rate }(=\bar{C} F)\end{array}$ \\
\hline F(C) & integral of $f(C)$ from $C$ to $\infty$, i.e., frequency of events above $C$ & $x_{i}$ & variable degree of initiating failure in category $i$ \\
\hline$F^{i}$ & frequency of initiator category $i$ & \&1...) & Dirac's $\delta$ function \\
\hline$F_{k}^{i}$ & $\begin{array}{l}\text { frequency of accidents of initiator category } i \text {, reaching the end } \\
\text { of phase } k \text { and thus entering phase } k+1\end{array}$ & $\begin{array}{l}\varepsilon_{C} \\
\varepsilon_{\eta}\end{array}$ & $\begin{array}{l}\text { lower value of "significant" consequences } \\
\text { lower value of "significant" caused failure }\end{array}$ \\
\hline$i$ & index of initiating categories & $\eta$ (general) & degree of caused failure \\
\hline j & index of parameters " $\mathrm{a}$ " & $\eta(C)$ & failure of degree $\eta$ as caused by a consequence (or damage) $C$ \\
\hline k & index of accident phases & $\eta\left(C ; a_{m+1}, \ldots, a_{n}\right)$ & $\eta(C)$ for specific parameters $a_{m+1}, \ldots, a_{n}$ \\
\hline MCA & $\begin{array}{l}\text { upper j value for parameters in consequence calculation } \\
\text { maximum credible accident }\end{array}$ & $\eta^{*}$ & $\begin{array}{l}\text { special consequence value in a simplified treatment without } \\
\text { parameter uncertainty }\end{array}$ \\
\hline
\end{tabular}




\title{
RISK-ASSESSMENT METHODOLOGY FOR FAST BREEDER REACTORS
}

by

Karl O. Ott

\begin{abstract}
The methods applied or proposed for risk assessment of nuclear reactors are reviewed, particularly with respect to their applicability for risk assessment of future commercial fast breeder reactors. All methods are based on the calculation of accident consequences for relatively few accident scenarios. The role and general impact of uncertainties in fastreactor accident analysis are discussed. This discussion shows the need for improvement of the methodology.

A generalized and improved risk-assessment methodology is outlined and proposed (accident-spectra-progression approach). The generalization consists primarily of an explicit treatment of uncertainties throughout the accident progression. The results of this method are obtained in form of consequence distributions. The width and shape of the distributions depend in part on the superposition of the uncertainties. The first moment of the consequence distribution gives an improved prediction of the "average" consequence. The higherconsequence moments can be used for consideration of risk aversion.
\end{abstract}

The assessment of the risk of one or a certain number of nuclear reactors can only provide an "isolated" risk assess ment. The general problem of safety risk assessment and its relation to public acceptance of certain modes of power production is a much broader problem area, which is also discussed.

\section{INTRODUCTION}

The technology of the exploitation of nuclear energy for the largescale production of electricity by means of thermal reactors was largely developed in the fifties and sixties. Although the use of nuclear energy as such was conceptually new, the development of reactors could, to a large extent, be based upon the experience with existing technologies. Cooling systems with pipes, pumps, vessels, and heat exchangers were not new. Rapid control of 
various reactions and processes had been used in other areas. Further, the entire production of electricity from hot steam is essentially independent of the source of primary energy.

However, there are essential differences in the use of nuclear energy as compared to the classical forms of energy that make nuclear reactors potentially more dangerous than other power plants. There are two basic reasons.

First, the nuclear fuel placed in a reactor suffices to maintain the energy production through chain reaction of nuclear fission for months. A direct consequence of this fact is that the level of energy production may accidentally be raised substantially since it is not constrained by lack of fuel.

Second, the fuel and part of the fission products are radioactive, having half-lives of years or centuries.

These major differences required, on the one hand, new technological developments, particularly in the areas of handling of fuel and fission products, shielding, and control of the power level. On the other hand, the potentially larger danger prompted an unparalleled emphasis on safety.

In the same sense as the technical development of the reactors is based on experience with existing technologies, also the development of the safety assessment is based upon experience with other systems. Failures of com. ponents, malfunctions, operator errors, errors in design or construction, etc., are common to all kinds of larger technical systems; so are types of possible consequences: damage to the plant or property damage off-site, and fatal or nonfatal injuries to personnel or to people outside the plant.

However, because of the possibly higher inherent hazard, much more stringent requirements have been developed for nuclear reactors than for other systems. These requirements are specified by the Nuclear Regulatory Commission in the form of standards and criteria.

The general goal of the safety requirements is to reduce the risk to the public so that it is smaller or much smaller than other risks to which the public is exposed. The "other risks" include acts of God, natural exposure to cosmic and nuclear radiation, and the health hazards of fossil-fueled power production.

Generally speaking, safety can never be absolute; the re will always be a finite risk involved in every man-made system or operation. Neither the knowledge nor the technological abilities can ever be absolutely complete. Therefore, the risk can never be made exactly zero, although it can be reduced considerably by appropriate measures. The finite risk that remains after a conscientious effort of risk reduction is called "residual risk" in the following. 
Two examples may illustrate the finite risk in otherwise beneficial operations:

1. The benefits and risks of major medical treatments or surgical operations are obvious. Also obvious is the "time dependence" of the risk: In the early phases of the development of a treatment or of a type of operation, the risk is to be reduced below a certain threshold prior to any widespread application. Tests on animals and in some cases on human beings, under specially controlled conditions, are conducted to show that the initial risk of a general application is acceptable. Nevertheless, the risk in early applications is generally larger than after a longer maturation period of the technique.

2. The power production in fossil-fueled (coal) power plants is a second example. Injuries to employees may occur during mining, transportation, and plant operation. The health hazard to the public results primarily from the effluents of the power plant. The quantitative evaluation of this latter hazard, e.g., in terms of life-span reduction measured in man-years per megajoule for a given plant, is hampered by the inadequacy of our knowledge in this a rea. The hazards of effluents of fossil-fueled power plants are not only due to chemical compounds (e.g., $\mathrm{SO}_{2}$ ) or particulates; the effluents also include significant amounts of radioactivity.

The strong emphasis on the safety of nuciear reactors led to the development of a multilevel safety concept. The general goal of this safety concept, the reduction of the residual risk to a comparatively very low and thus acceptable value, is achieved by measures on three levels.

The first level includes redundancy in the design, quality assurance in the construction, and proper monitoring of essential components and functions during the operation. The second level comprises the measures to cope with all kinds of specifically anticipated or generically defined incidents. The third level includes the safety-design measures that are supposed to become effective in cases of major failures.

One can strongly reduce the risk involved in nuclear power plants by the measures indicated above without having a quantitative evaluation of the residual risk. Although a reliance on unquantified risk is far from being ideal, it is the internationaliy accepted judgment that the risk reduction achieved in this way for thermal power reactors is sufficient to allow large-scale application of thermal reactors, especially LWR's. (Reference 1 fully supports this judgment by a quantitative evaluation.)

To obtain a better foundation of this judgment as well as for further reduction of the residual risk, it is necessary to develop and apply a quantitative safety-assessment method. Thus, in addition to the general goal of safety requirements, i.e., the risk reduction as such, one identifies a second goal of safety considerations: the quantitative assessment of the residual risk. 
Due to the nature of the safety problem, this quantitative riskassessment method is by necessity a probabilistic analysis. The discussion of probabilistic safety analysis, particularly for fast reactors, is the main subject of this report.

It is not meaningful and sensible to consider the accidental risk from nuclear reactors in isolation. The risk from nuclear reactors must be seen in relation to the risks resulting, on the one hand, from all other types of accidents, and on the other, from substitutions by other types of power stations or from nonavailability of needed energy. An excellent qualitative discussion as well as extensive quantitative risk comparisons are presented in Ref. 1. Risk comparisons are also discussed here, but from a different perspective. Safety and risk assessments and their comparisons are viewed in their longterm evolution (see Sec. III). 


\section{SURVEY OF SAFETY AND RISK-ASSESSMENT METHODS}

In all approaches or methods to assess the safety of any system, one considers the consequences of accidents as well as the probabilities of their occurrences. Differences in the approaches are mostly due to the different emphasis on consequences versus probabilities. In one class of approaches, one considers explicitly only the consequences; accident probabilities are considered only implicitly through judgment about the "credibility" of various accidents. These approaches are categorized in the following as "consequenceoriented approaches." If, in addition to accident consequences, the accident probabilities are considered explicitly, one can evaluate the risk, i.e., the product of consequence and probability. This leads to "risk-oriented approaches."

As indicated in Sec. I, the consideration of safety has two basic goals. The major goals are to make a plant safe (or safer than previous ones) and to assess the residual risk. The subject of this report is only the assessment of the residual risk. The application of probabilistic analysis for actual risk reduction is only briefly discussed in Sec. II.C. 1 (see Fig. 5 later).

Safety considerations are principally concerned with the safety on-site and off-site. The problem of on-site safety of nuclear reactors is in principle not different from the safety of other complicated plants where malfunctions or failures may lead to plant damage or to injuries of personnel. The unparalleled emphasis on safety is directed toward the protection of the populace, i.e., toward the prevention of the propagation of accidents beyond the plant boundary. Therefore, in the evaluation of the residual risk, one concentrates on accidents that may have a chance for an of

The survey of safety and risk-assessment methods is presented under a unifying perspective; the historical development of assessment methods is viewed as a step-by-step reduction of the individual judgmental component in the assessment. The generalized methodology proposed here for fast-reactor application represents a further step in the reduction of the impact of judgment on safety assessment.

\section{A. Consequence-oriented Approaches}

\section{The Maximum-credible-accident (MCA) Approach}

The first formalized approach to judge the safety of nuclear reactors and to provide a basis for devising the protective system was the maximum-credible-accident (MCA) approach. Engineering judgment was used to survey possible accidents or accident categories. Based on an intuitive estimate of their probabilities, the set of surveyed accidents was subdivided into credible and noncredible accidents, the latter category containing events of low probability. However, the accidents that were termed noncredible were 
not completely disregarded. The protective system was generally designed in such a way that it provided a wide safety margin so that many of the accidents that are not termed credible can still be contained.

The weakness of this first approach appears to be the intuitive and judgmental nature of the subdivision between credible and noncredible occurrences. However, the main weakness of the MCA approach is that the actual safety limits of the reactor plant consist of the undefined margin of safety that is based on individual judgment, which could not be critically challenged, since the approach did not include the disclosure of the design basis.

\section{The Design-basis-accident (DBA) Approach}

The major disadvantage of the MCA approach was removed by the transition to the design-basis-accident (DBA) approach. Instead of the unspecified margin of safety that is built into the protective system for accidents beyond the MCA, one investigates in detail low-probability accidents with major consequences as the explicit basis for the design (DBA's). In this way, one obtains specific information about what kind of accidents a given reactor and safety-system design can cope with. Furthermore, the somewhat individual judgment of the credibility of accidents is avoided.

Nevertheless, the DBA approach is also based upon best engineering judgment about accidents to be included or not included in the design basis. The judgment in the DBA approach is generally concerned with accidents of a much lower probability than those often considered as upper limit in the MCA approach. Furthermore, the judgment in the DBA approach is applied to a specific set of accidents that have been widely discussed, nationally and internationally. Therefore, the judgment about the inclusion of a type of accident into the design basis is of the character of a "collective" best engineering judgment and is therefore different from the more individual judgments in the early applications of the MCA approach.

The DBA is part of the multilayered framework of safety concepts developed by the Nuclear Regulatory Commission as a basis for reactor licensing.

The occurrence of a major accident requires a very unlikely sequence of failures. It is even more unlikely that accidents reach beyond the "envelope" provided by a set of DBA's. Nevertheless, there is the principal possibility, although with a very small probability, that vital parts of the protective system fail, or that consequences of supposedly contained accidents are larger than calculated. This leads to the "residual" $r i s k$, which remains unquantified in the DBA approach. 


\section{B. Concepts of Risk-oriented (Probabilistic) Approaches}

\section{General Considerations}

The major disadvantages of the DBA approach are the lack of quantification of the residuai risk and the use of judgment as basis for selecting the DBA's. The risk-oriented approaches try to remove both disadvantages. Occurrence rates or annual probabilities are assigned to the various accidents. The use of quantitative probabilities provides a more appropriate set of DBA's. There is no need to disregard accidents with a very low probability. A basic part of the probabilistic approach is the quantification of the residual risk by combining accident probabilities and consequence predictions properly.

In the light of these obvious advantages, one may ask "Why is the probabilistic approach not in widespread use for analyzing the safety of nuclear reactors?" The major reason has been the uncertainty of the probability values one has to assign to the individual or correlated failure of components, of operator errors, etc. This reason for not including probatilities explicitly into the safety assessment may have applied in the past. However, in recent years, data on failure rates of components and other input for a probabilistic analysis have been collected, and methods to quantify system failures have been developed. Nevertheless, sizable uncertainties still exist (e.g., see Fig. 3 later).

Since there is considerably less experience available for fast reactors than for thermal reactors, and since fast-reactor accidents would in most cases exhibit a physically more complicated progression than thermalreactor accidents, the uncertainties that enter the probabilistic assessment of fast-reactor safety are substantially largex than those for thermal reactors. Therefore, an elaborate probabilistic approach for fast reactors should include the detailed treatment of the uncertainties, particularly in the prediction of the consequences.

The assessment of reactor safety by a probabilistic approach was proposed in the United States as early as 1962 by Thompson. ${ }^{2}$ In 1967, Farmer ${ }^{3}$ introduced a probabilistic approach for the analysis of the safety of nuclear reactors, which was adopted in the UK as a basis for licensing. The application of the same approach to fast reactors was proposed by Farmer and Gilby in Ref. 4.

In recent years, several publications on probabilistic reactorsafety assessment (for thermal and for fast reactors) have appeared in the literature, and more and more countries are including probabilistic approaches into their licensing procedure. The most extensive study of the safety of commercial LWR's as investigated by a probabilistic approach was carried out in the USA under the direction of Norman Rasmussen. 'The Rasmussen study represents a substantial leap forward in the methodology, and in its thoroughness and completeness compared to all other available studies of LWR safety. 
The various probabilistic approaches that have been used or studied in recent years will be briefly reviewed in this report. A generalized probabilistic approach will be outlined (Sec. III), since the available methods do not appear to be satisfactory for fast-reactor applications.

\section{Farmer's Limit-line Approach}

The conceptual idea of Farmer's approach ${ }^{3}$ is to reduce the risk of individual types of reactor accidents below a limit that may be considered acceptable. Thus, the conceptual basis of this approach is a so-called "limit line," which serves as a criterion of the acceptability of the risk of any type of a reactor accident. If the evaluation of the risk of a type of an accident leads to a value above the risk criterion, design changes are required to bring this risk below the limit line.

Farmer applied, as a measure of the risk to the public, the amount of ${ }^{131}$ I released in an accident. Other radioactivity releases can be expressed in equivalent ${ }^{131} \mathrm{I}$ amounts, where the equivalence is defined on the basis of the same health hazard. Because of the application of radioactivity release as a measure of the risk, Farmer also calls the risk limit line the "release criterion."

Figure 1 depicts Farmer's limit line, $\tilde{f}^{F}(C)$, in terms of accident probability per year as a function of curies of ${ }^{131} \mathrm{I}$ release per accident, C.

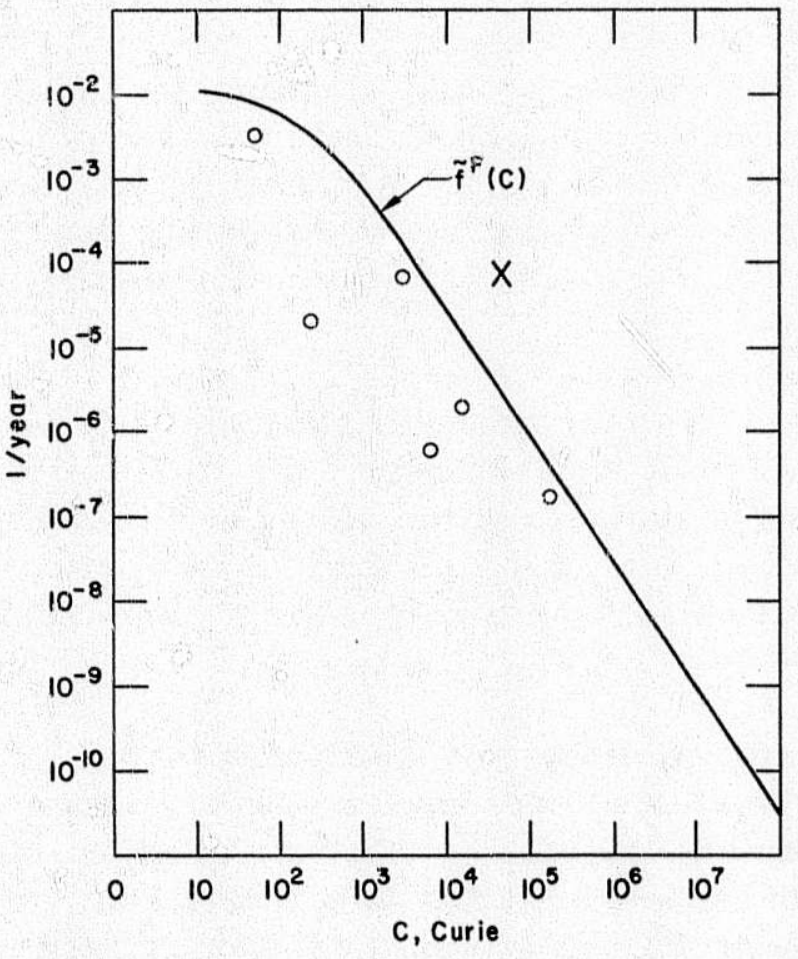

Both ordinate and abscissa are given in a logarithmic scale. The circles represent accident risks below the limit line. The cross shows an unacceptable risk, which must be reduced through appropriate design changes.

The shape of $\tilde{f}^{F}(C)$ is constructed on the basis of the following two considerations: A risk resulting from a few events with large consequences is generally felt less acceptable than the same risk resulting from many small consequence events. (For example, a large airplane crash is less "acceptable" than mariy car accidents with the same number of fatalities.) Therefore, $\tilde{f}^{\mathrm{F}}(\mathrm{C})$ should asymptotically decrease more strongly than 1/C with increasing C. Farmer chose the relationship

Fig. 1. Farmer's Limit Line or Release Criterion ${ }^{3}$

$$
\tilde{f}_{a s}^{F}(C) \propto C^{-3 / 2}
$$


to reflect the public aversion to high risks. Extrapolating this $C^{-3 / 2}$ curve, as shown in Fig. 1, to low-consequence values yields far too high probabilities for small consequences to be economically tolerable. (For example, accidents with a relea se around $10 \mathrm{C}$ would appear to be "acceptable" according to the extrapolation, with a frequency of several accidents per year.) To disallow too frequent incidents with low release, Farmer flattened his release criterion at low curie values.

The magnitude of the release criterion was fixed by a consideration involving the total risk: The individual values (circles in Fig. 1) to be compared with the release criterion represent types of accidents such as loss of flow ( $L O F$, due to loss of power to the pumps), pipe rupture, and transient overpower (TOP, due to accidental insertion of reactivity), with scram action assumed to fail in all cases. There are only a few of these types of accidents, particularly of those yielding a risk value close to the release criterion. For an urban population of 4 million people, one would obtain from typically four or five points near the curve* 5-10 cases of thyroid cancer per $10^{3}$ reactor years, or about 0.01 case per reactor year. Among the 4 million people, the natural incidence rate is about 72 cases per year. Thus, the additional risk from a reactor located near a city of 4 million people is only about $10^{-4}$ of the natural incidence rate. Considering this to be acceptable as an upper limit determines then the magnitude of the curve in Fig. 1.

\section{Accident-risk Envelopes}

A concept that is seemingly related to, but is logically different from Farmer's limit line, is the "accident-risk envelope," denoted here by

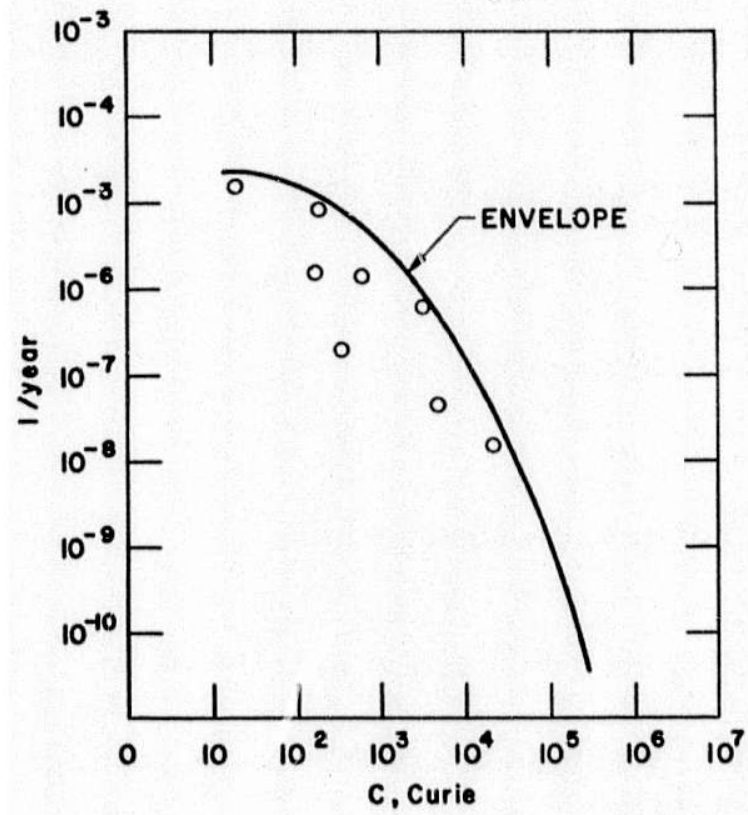

Fig. 2. Illustration of a Risk Envelope
$\hat{f}(C)$. It is obtained by calculating a number of frequency-versus-consequence points, plotting them in an f-versus-C diagram, and drawing a smooth envelope above these points. The rationale behind this concept is that, although the number of explicitly treated accident scenarios is not complete, they probably suffice to stake out the upper part of the area in the f-versus-C diagram in which the various f-versus-C values may be found. Thus, the envelope should provide an upper limit (see Fig. 2).

The logical difference between the release criterion and the accident-risk envelope is that the criterion is set a priori, whereas the envelope is drawn a posteriori (i.e., after the accident analysis). This logical difference appears

*With the magnitude shown in Fig. 1. 
clearly in the application. Both methods may use the same analysis of accidents, and the results may be depicted in the same diagram. If one or more of the f-versus-C points are above the release criterion, design modifications are required to bring these points below the criterion. If the points are used to draw an envelope, no direct requirements for design change result; the envelope is drawn above all points. In practice, however, one will clearly pay appropriate attention to a special accident if its risk should be well above an envelope resulting from the other events.

\section{Probability Distributions of Consequences}

The general mathematical concept of the simultaneous description of accident probabilities and consequences* is the probability distribution of the consequences, $\mathrm{p}_{\mathrm{C}}(\mathrm{C})$, or the corresponding frequency distribution of the consequences, $f(C)$. The quantity $f(C) d C$ describes the frequency of accidents with consequences $C$ in $\mathrm{dC}$, measured in events per year per $C$ unit. The frequency distribution $\mathrm{f}(\mathrm{C})$ is the sum of the individual contributions of all the accidents considered and is related to the consequence distribution by

$$
p_{C}(C)=\frac{1}{F} f(C)=\text { probability distribution of consequences, }
$$

with

$$
F=\int_{\text {all } C} f(C) d C=\text { frequency or annual accident probability. }
$$

The following integral concepts are needed in the evaluation and assessment:

$$
\begin{aligned}
& \bar{C}=\frac{1}{F} \int_{\text {all } C} C f(C) d C=\text { average consequence per accident; } \\
& \bar{R}=\overline{C F}=\int_{\text {all } C} C f(C) d C=\text { average annual risk; } \\
& \overline{C^{2}}=\frac{1}{F} \int_{\text {all } C} C^{2} f(C) d C=\text { average consequence square per } \\
& \text { accident. }
\end{aligned}
$$

An approach based on consequence distributions is described briefly in Sec. IV.

An instructive function that can be derived from the consequence distribution is the corresponding indefinite integral

$$
F(C)=\int_{C}^{\infty} f\left(C^{\prime}\right) d C^{\prime}=\text { frequency of accidents larger than } C,
$$

\footnotetext{
"Consequences" may be defined and evaluated at various stages of the accident progression.
} 
where $f(C)$ represents the differential* and $F(C)$ the integral* consequence distribution. This integral distribution is used in the Rasmussen study for the description of the final results.

\section{The Rasmussen Approach}

The Rasmussen study ${ }^{1}$ of LWR safety is the first extensive safety study that conceptually aims at the probability distribution of consequences and at average quantities derived from consequence distributions, Eqs. 2 . Consequences at a certain intermediate stage of the accident progression corsist of the release of radioactivity from the containment. In the Rasmussen study, the release of radioactivity is subdivided in "release categories," and the results are depicted in the form of a histogram (see Fig. 3). In the light of the uncertainties involved in calculating consequences and in finding the accident probabilities, a histogram of the type of Fig. 3 may be fully appropriate.

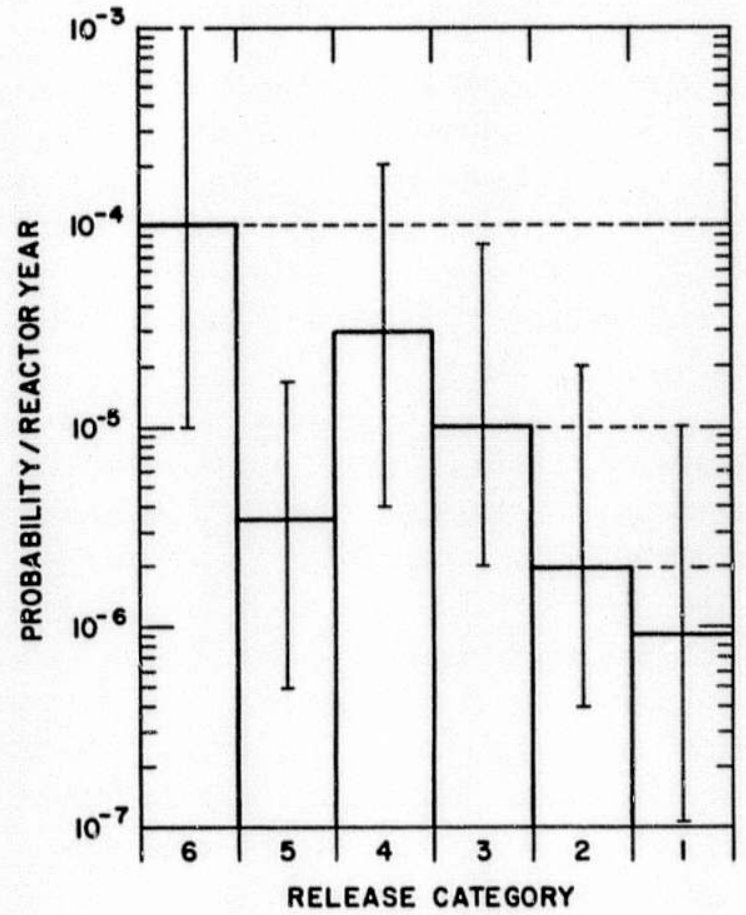

Fig. 3

Release Frequency vs Release Categories (p. 136 of Ref. 1). The relation of release categories and release is given on p. 118 of Ref. 1. The error bars represent a $90 \%$ confidence range.

The propagation of the released radioactivity and the resulting exposure of the populace is analyzed in Ref. 1 in great detail. Experimental weather distributions and detailed population distributions have been used. The result then consists of a detailed histogram of the distributions of eventual consequences (such as acute illness, latent cancer, or fatalities). Actually presented in Ref. 1 are the corresponding integral distributions $F(C)$. The $F(C)$ as they result from such a detailed treatment of the radioactivity propagation are practically smooth functions. 
The conceptual treatment of the cause-consequence relation in the Rasmussen approach is discussed below.

\section{Limit-line and Envelope Integrations}

The number of investigated individual accidents is naturally limited. However, it is generally assumed that one is able to identify the major types of accident contributions to the total risk through the process of "collective" best engineering judgment. Therefore, Farmer ${ }^{3}$ found it justified to derive the magnitude of his release criterion from four or five major contributors. This as sumption has been fully verified in Ref. 1. The systematic search for possible accident progressions along discrete paths led originally to a large number of possible discrete accident paths. The evaluation of the corresponding probabilities and consequences showed that only a few of all possible paths were major contributors to the total risk. Thus, the Rasmussen study supports in detail the prevailing as sumption.

The opposite approach consists of assuming that the relatively broad accident categories can be resolved into very specific and detailed accident scenarios, much more detailed than in the Rasmussen study. One then expects that the complete description consists of many specific scenarios. The few cases actually calculated are used to construct a continuous variety by drawing an "envelope" (see Sec. II.B.3). Similarly, Farmer's release criterion might also be considered as an enveloping upper lirnit.

Some authors (e.g., Bell ${ }^{5}$ and Otway and Erdmann ${ }^{6}$ ) propose to find the risk from "all" accidents by integration over the limit line or the envelope. However, the curves obtained either through a priori definition [Farmer's limit line $\tilde{f}^{F}(C)$ ] or through construction as an envelope, say $\hat{f}(C)$, are not "actual" distributions. "Actual" in this context indicates that the distribution has the correct conceptual meaning of a probability distribution of the consequences, even though it may not be quantitatively correct. The limit line and the envelopes are only substitutes for distributions. These substitutes should not be directly inserted into Eqs. 2 to find the probability and the average consequence.

Since $\tilde{f} F(C)$ and $\hat{f}(C)$ are limiting or enveloping individual events, their dimension is "frequency per event":

$$
\operatorname{dim}[\tilde{f} F(C) \text { or } \hat{f}(C)]=\frac{\text { frequency }}{\text { event at } C}
$$

For $\tilde{f} F(C)$ or $\hat{f}(C)$ to be amenable to integration, they must be multiplied with density functions, $\rho F(C)$ or $\hat{p}(C)$, describing the number of events per $C$ unit:

$$
\operatorname{dim}[\rho F(C) \text { or } \hat{\rho}(C)]=\frac{\text { events at } C}{C \text { unit }} .
$$


Only the product of Eqs. $3 \mathrm{a}$ and $3 \mathrm{~b}$ represents conceptually a consequence distribution with the dimension frequency per $C$ unit, which may then be inserted into Eqs. 2; e.g.,

$$
\begin{aligned}
& \int \rho F(C) \tilde{f}^{F}(C) d C=F^{F} ; \\
& \int C \rho F(C) \tilde{f} F(C) d C=R^{F} ; \\
& \int \hat{\rho}(C) \hat{f}(C) d C=\hat{F} ; \\
& \int C \hat{\rho}(C) \hat{f}(C) d C=\hat{R} .
\end{aligned}
$$

Bell ${ }^{5}$ chose the density function $\rho F(C)$ in such a way that it is constant $(=1)$ along the logarithmic consequence axis:*

$$
\rho F(C) d C=d \log C=\frac{2.30}{C} d C,
$$

with log denoting Briggsian logarithm. This gives for Eqs. 4

$$
\int \frac{2.30}{C} \tilde{f} F(C) d C=F^{F}
$$

and

$$
\int \frac{2.30}{C} C \tilde{f}^{F}(C) d C=R^{F} \text {. }
$$

Whatever the choice of a density function, it involves by necessity a high degree of arbitrariness. For example, the as sumption of a constant density distribution along the linear scale instead of a constant over the logarithmic scale yields a value for $\overline{\mathrm{C}}$ about 300 times as large as given by Eq. $7 \mathrm{~b}$ if the integration is extended over the range of Fig. 1. However, with a constant-density disiribution over the linear scale, one assumes, e.g., jetween $\mathrm{C}=10^{6}$ and $10^{7} \mathrm{Ci}, 100$ times as many accidents than between $10^{4}$ and $10^{5} \mathrm{Ci}$, which does not seem sensible. Equation 6 seems to be more platisible, although both density functions are arbitrary.

The envelope-integration approach as actually applied by Otway and Erdmann ${ }^{6}$ is not the same as outlined in Eqs. 5. Otway and Erdmann plotted the inverse results (i.e., ${ }^{131}$ I release versus annual probability) and found, in principle, first the inverse function $C(f)$ :

$$
\mathrm{C}^{\mathrm{OE}}(\hat{\mathrm{f}})=\text { inverse of } \hat{f}^{\mathrm{OE}}(\mathrm{C})
$$

*A similar choice might be made for the envelope integration. 
Then the function $C^{O E}(\hat{f})$ was integrated with respect to $\hat{f}(\hat{f}$ is denoted by $P$ in Ref. 6). The inverted consequence distribution $C(\hat{f})$ was multipled by a factor $m_{t} / \mathrm{wb}$ (mortality probability per rem of radiation) and integrated with repect to $\hat{f}$ :

$$
m_{t / w b} \int C^{O E}(\hat{f}) d \hat{f}
$$

The quantity that results from the integration 9 was applied to find a "total risk."

This approach appears to be highly questionable.* The concept "total risk" cannot be obtained from a consequence distribution. Probabilistic analysis yields an "average" risk or "average" consequences, as defined by Eqs. 2. The integrations may, if so desired, be carried out over the inverse function $C(f)$ instead of $f(C)$; e.g.,

$$
\begin{aligned}
& \int_{\text {all } C} f d C=\int_{\text {all } f} f \frac{d C}{d f} d f=F ; \\
& \frac{1}{F} \int_{\text {all } f} C(f) \cdot f \frac{d C}{d f} d f=\bar{C} .
\end{aligned}
$$

The results of Eqs. 10, however, are conceptually and quantitatively the same as the results of Eqs. 2; i.e., the integra: Eq. $10 a^{* *}$ gives $\mathrm{F}$, and the integral in Eq. $10 \mathrm{~b}$ gives $\overline{\mathrm{C}}$.

It is believed that, due to the arbitrariness involved in the density functions, the approach to estimate the combined risk of "all" accidents by limit-line or envelope integration is inappropriate and should not be applied.

C. Description of the Cause-Consequence Relation

For any type of quantification of the residual risk one needs a description of the relation between accident initiations (causes) and the eventual (or intermediate) consequences. These "cause-consequence relations" may be illustrated and described principally in two ways: in the forward direction (i.e., by following the physical progression) or in the backward direction (i.e., by investigating the successive causes in a branched chain of events).

\section{The Event-tree Description}

The first version of a forward description of a sequence of events involving branching points was the so-called "decision tree," a technique to

*Compare also Farmer's remark on this approach in Ref. 7.

**Integrating the middle integral in Eq. 10a by parts yields an integral over the inverse distribution $C(f) d f$ which apparently has been used in Eq. 5 of Ref. 6 (see Eq. 9 here). 
explore systematically the expected consequences of a succession of decisions. Forward description of accident progression has also been applied in the Rasmussen study ${ }^{1}$ of the safety of LWR's. The term "event tree" was coined in this study for the formal description of the accident progression through a sequence of failures. Figure 4 is an example of an event tree (from p. 89 of Ref. 1). Probabilities are assigned to the various events as a basis for subsequent quantitative evaluation.

The event-tree technique provides also an excellent basis for the incorporation of the interactions between component failures or subsystem functions. The incorporation between these interactions strongly reduces the original straightforward event tree (see Fig. 4). The resulting reduced event tree is then the desired descriptive framework for the accident risk as ses sment.*

\section{The Fault-tree Description}

The backward description of a combination of failures is the famous "fault-tree" technique, which systematically reduces the failure of a complicated system to failures of the components of the system. A huge body of literature exists on the reliability of components and on the evaluation of fault trees.

The basic feature of a fault tree is a specified failure event of the system, the so-called "top" event. The direct conditions for occurrence of the top event represent the next level of the fault tree. The fault tree is developed further until all branches are reduced to failures of the components of the system. Mostly, components are assumed to have a binary response; i.e., they either function or fail to function as expected.

The applicability of the fault-tree technique to safety analysis is very limited, primarily for two reascns. First, there is no well-defined "top" event; rather, there is a large and continuous variety of eventual consequences. Second, the forward instead of a backward description is to be preferred in safety analyses, since large phases of the accident progression are mathematically described by (complicated) initial-value problems. The mathematical methods for solving initial-value problems in the forward direction are well developed, but not for solving these problems backwards.

The fault-tree technique is, however, very useful for estimating well-defined failure probabilities of subsystems (e.g., failure of scram). Fault trees have been extensively used for these purposes in the Rasmussen ${ }^{1}$ study and in other studies (e.g., Ref. 8). Component failure probabilities are often subject to large uncertainties (sometimes by a factor of 10 or more in both directions). These uncertainty bands must be consistently carried through

*The event-tree reduction results in the elimination of practically irrelevant branching points (see Fig. 4). 


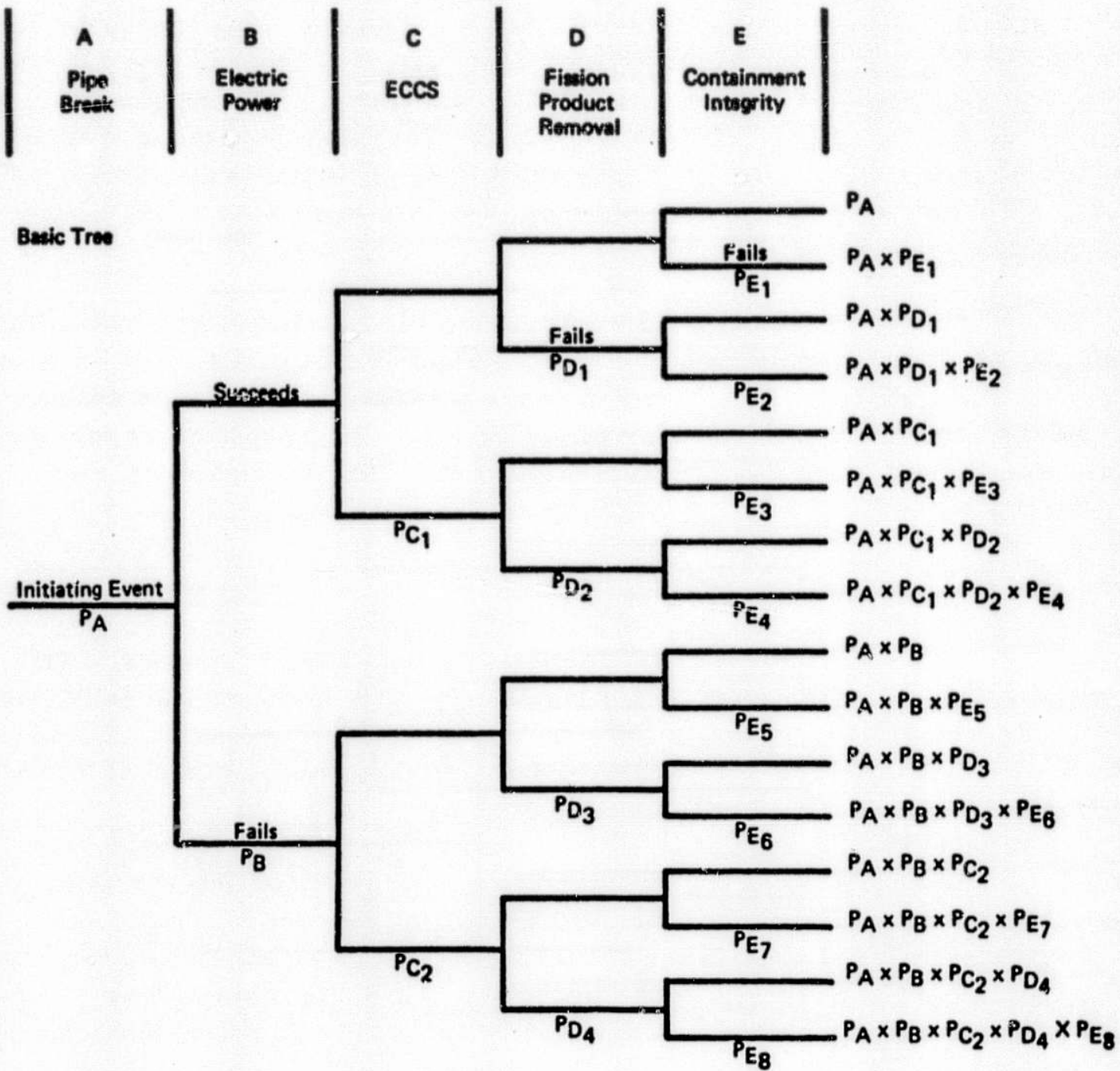

Reduced Tree

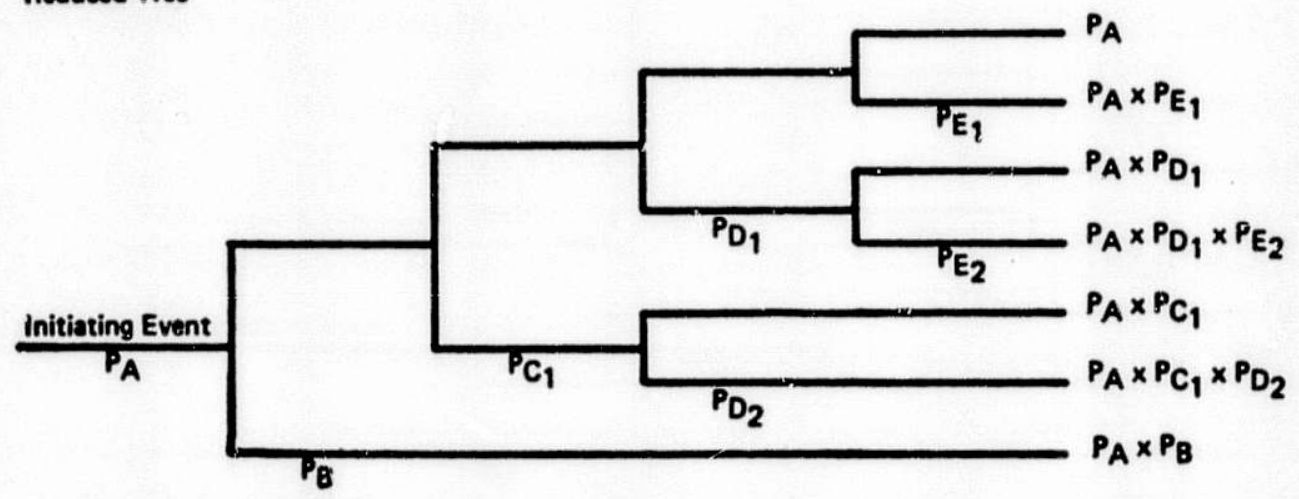

Now - Sines the probebility of failure, $P_{0}$ is generally less than $C_{1} 1$, the probability of eucoes (1.P) is always clow to 1. Thus, the probability aseociated with the upper (avecen) brenches in the tres is enesimed to be 1.

Fig. 4. Simplified Event Trees for Large LOCA (p. 89 of Ref. 1) 
the fault-tree evaluation and are eventually reflected in a corresponding uncertainty of the subsystem failure as calculated by the fault-tree analysis. ${ }^{1}$ The fault-tree technique for the evaluation of subsystem failures seems far from ideal. According to Rasmussen, ${ }^{9}$ it is cumbersome, expensive, and not unique. (That is, different people may draw somewhat different fault trees.) Therefore, a simpler and more efficient method is desirable. ${ }^{9}$

\section{Accident-progression Diagrams}

The forward description of an accident progression is extensively applied to fast-reactor safety in the so-called "Safety-assurance Diagrams" or "Accident-progression Diagrams." Figure 5 (from Ref. 10) shows an

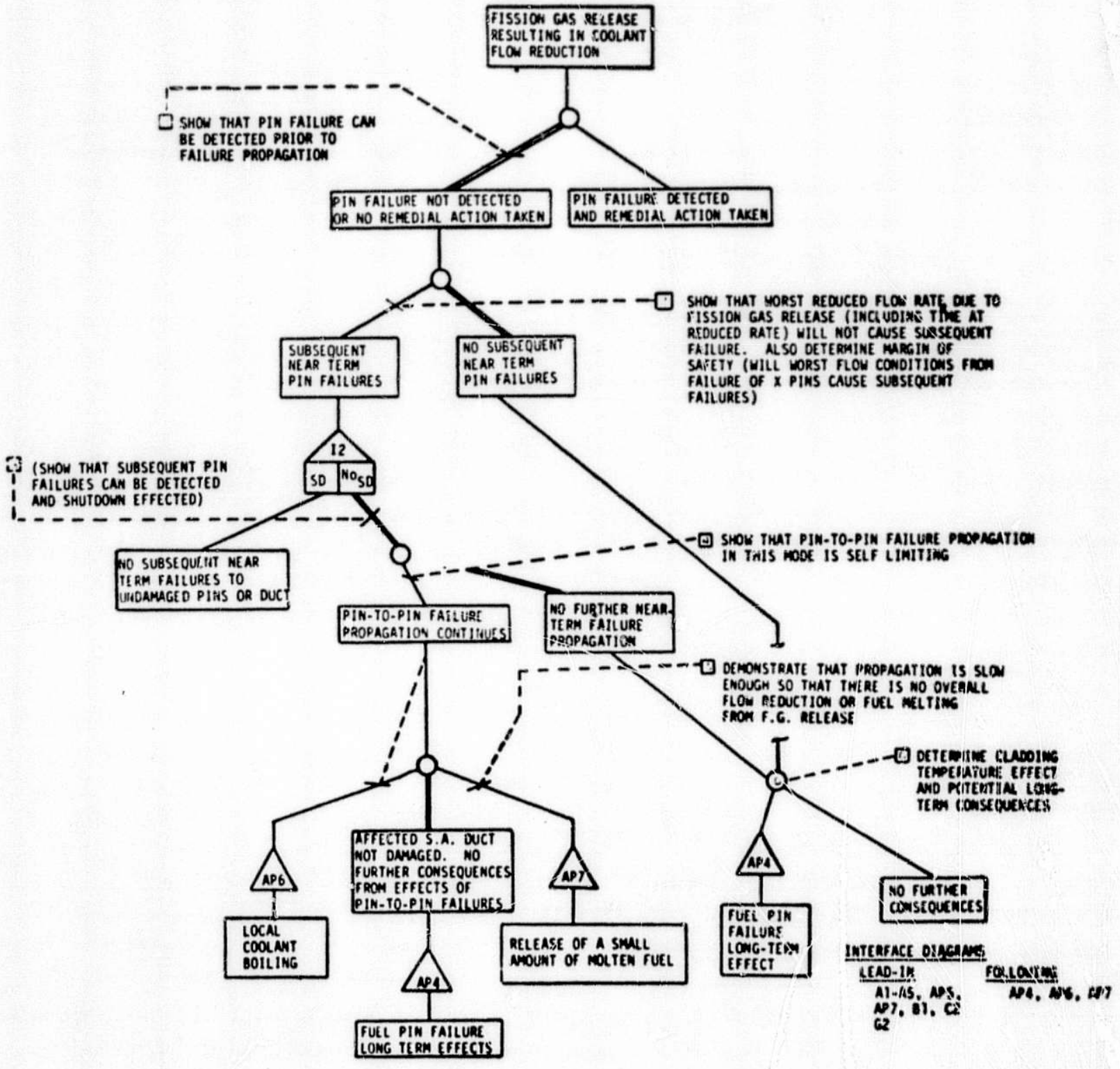

Fig. 5. Example of an Accident-progression Diagram 
example of an accident-progression diagram and of the qualitative way it is applied to improve or ensure the safety of the plant.

The more likely path emerging from a branching point is drawn as a double line, the less likely one as a single line. Special guidance for the design of the plant is derived from the accident-progression diagram, such as:
a. Make sure that the progression along this (less likely) path is very unlikely, or
b. Make sure that the progression along this (more likely) path can be detected and a shutdown device can be triggered.

The safety of the plant can be improved by carefully implementing the instructions derived from the accident-progression diagrams. However, the number of such instructions is quite numerous, and it is not obvious how to distribute the emphasis over the very large task. This has been pointed out by El-Sheikh, ${ }^{11}$ who developed a semiquantitative scheme for ordering accident-progression diagrams in a "cause-effect matrix."

\section{Simulation Languages and Computer Models}

Simulation of the behavior of complicated systems is a general problem. Simulating the behavior of a system, especially its development with time, consists of investigating a "model" of the system rather than the systern itself. In many cases this model can only consist of a "computer model" in which the behavior of the system is described by appropriate mathematical operations. The investigation of the variety of accidental behavior of nuclear reactors is a typical example for which a cornputer model is the only practical tool. However, any individual phenomena that may occur during accidents must be investigated by means of actual "physical models" in order to obtain the specific physics information required for the construction of appropriate physical models.

The methodology of simulation by computers is well developed. Fairly general, and thus widely applicable, computer models have been developed in the recent decade (simulation languages). However, the complexity of the safety analysis of nuclear reactors, especially of fast reactors, practically precludes the application of one of these general simulation languages; rather, it requires the development of highly specialized computer models for the description of the special complexity and variety involved in accidental reactor behavior.

The safety-analysis system SAS (e.g., Refs. 12 and 13) is a detailed computer model for the analysis of the main accident categories to be considered in fast-reactor safety analysis. However, the long computer time of SAS precludes its application to the investigation of the broad variety of accidents, which results from the inaccuracies in data, lumped parameters, and 
some of the computer models. Therefore, the development of a special, fastrunning program, PARSEC (Parametric Safety Evaluation Code), has been started at ANL in cooperation with Purdue University.

D. The Accident-spectra-progression Approach

1. The Principal Impact of Uncertainties

The description of the cause-consequence relation by an event tree or by an accident-progression schematic (see Sec. IV) provides a coordinated overview of the possible accident-progression paths. Evaluation requires the treatment or simulation of the accident progression by an appropriate computer model, which in itself must be based on actual experimental modeling of the important physical features.

If the computer model would exactly simulate the accident progression that follows a precisely defined initiating failure, the result would be a deterministic path for a given "initial" state of the reactor. For a continuously varying degree of an initiating failure (for example, degree of pipe rupture) and for a continuously varying state of the reactor (for example, through burnup) at the time of the accident, one obtains a continuous spectrum of deterministic accident-progression paths.

However, the analysis of major fast-reactor accidents is so complicated that inaccuracies in the predictions are unavoidable. Several positivefeedback phenomena may play a role during a fast-reactor accident; the resulting accident progression may be quite complicated, and certain inaccuracies are encountered in the description. Furthermore, there is no practical experience in such accidents, and no practical experience can be expected, since the probability of such accidents is made extremely small. Thus, the safety analysis has to live with substantial inaccuracies, although the uncertainty range will gradually be narrowed by further intensive research in FBR safety.

The nonlinearity of sensitivities of consequences is extremely large if a threshold type of phenomenon is involved (such as rupture of a vessel). Suppose the "expected" value of a pressure loading is below a threshold and will have no effect. A variation on one side then leads to zero effect, but the same variation on the other side may exceed the threshold. If the full distribution of the pressure loading is considered, some part of the "upper wing" may exceed the threshold. This example shows that the finite probability for exceeding the threshold may only be obtained by investigating the accident spectrum that contains the full distribution of the pressure loading.

The discussion of the principal impact of uncertainties shows that, if the prediction of the accident progression is hampered by substantial uncertainties, as is the case for the analysis of major accidents in large fast reactors, it is important to explicitly incorporate the uncertainties in the safety 
evaluation. An improved approach for accident analysis that explicitly includes the treatment of uncertainties is briefly outlined in the following. This approach is called the Accident-spectra-progression (ASP) approach, since a welldefined accident path is smeared out into a "spectrum" of accident paths if uncertainties are incorporated. A more detailed outline is presented in Sec. IV.

\section{Brief Outline of Accident-spectra-progression Approach}

The progression of severe fast-reactor accidents can be subdivided into a number of phases, such as (1) the accident initiation phase, (2) the multichannel phase, in which the subassembly structure is still intact, (3) the transition phase, in which the subassembly structure is deformed, and possibly (4) a disassembly phase. The nuclear accident is terminated in one of these phases. The further behavior of the system and the fuel is described in several additional phases, which include the behavior of vessel and containment, the postaccident heat removal, and the various radioactivity-removal procedures. If radioactivity should be released from the containment, its further propagation and the resulting exposure to the populace must be treated. The treatment of the radioactivity outside of the containment is practically identical for fastand thermal-reactor accidents. Therefore the detailed methods developed in the Rasmussen study can directly be taken over for these phases. However, the progression of accidents within the containment requires a different treatment from that in Ref. 1.

The uncertainties that hamper the predictions of accident progression may be clivided into three different types: first, the uncertainty of parameters and coefficients involved in the theoretical treatment; second, the uncertainty of special physical phenomena; and third, the inaccuracy of theoretical models due to approximations introduced in devising the model. The first two types of inaccuracies are the more important ones. The last one is more amenable to improvement if the resulting additional accuracy is really needed.

Uncertainties of parameters and coefficients may be expressed in the form of a probability distribution for that quantity, say $p_{a_{k}}\left(a_{k}\right)$, $a_{k}$ being one of the parameters. It is also required for the evaluation of the impact of the uncertainties of physical models to express these uncertainties as a variation of specially introduced coefficients.

The classical procedure for exploring the impact of uncertainties in a computational model is the sensitivity analysis, i.e., the variation of all important parameters and the computation of the resulting impact. To do this for fast-reactor accident analysis, codes for parametric safety evaluation are required for all accident phases. A first version of such a code (PARSEC, Sec. II.C.4 above) is being developed for the multichannel-accident phase. The application of these codes of each phase yields the "unweighted" results of a sensitivity study. These results are subsequently averaged with the applicable 
probability distributions as weights to produce the relevant consequence distributions at the end of each phase. This averaging represents a contraction of the detailed information of the sensitivity study. Only a reduced amount of information needs to be carried into the next phase. The application of this procedure will yield a distribution of radioactivity release from the containment, i.e., a more detailed distribution than that depected in Fig. 3. As indicated above, the further treatment can be the same as in the Rasmussen study.

In an explicit treatment of uncertainty distributions, a single accident path is resolved into a spectrum of accidents progressing through the various phases: ASP approach. A more detailed description of the ASP approach is presented in Sec. IV. 


\section{SAFETY AS EVOLUTION}

The consideration of safety and risk of many technological and other developments inherently involves two components. One is the quantification of safety and risk. The other comprises all considerations related to the question of what represents an acceptable risk or "How safe is safe enough." Both components are generally related, and they act upon each other. For some developments the interrelation is barely noticeable. For the development of nuclear power reactors, especially of the fast-breeder-reactor type, the interrelation of the two components of risk is stronger than for most other technological developments. Therefore, the considerations concerning the acceptability of the risks of nuclear power are discussed in the following.

This strong relationship between the quantification and acceptability of risk led to an unprecedented emphasis on safety during the entire development of commercial nuclear power. However, due to the complexity of the problem, the accuracy of the quantification still needs to be improved, especially for fast reactors. This lack of fully accurate quantification has been the basis for many controversies about nuclear power. Controversies naturally thrive on inaccuracy, on misunderstanding, and on unavailability of information. Further amplification of controversies results from those components of the riskacceptability problem that can hardly be quantified, since they are psychological or judgmental in nature.

The assessment of the risk from conceivable or hypothetical accidents of future fast breeder reactors represents a substantial part of the riskacceptance question. The methodology of this risk assessment, particularly the impact of uncertainties on the quantitative result, has been discussed above. This section is devoted to the application of accidental-risk-assessment results in a larger framework of an overall risk assessment. Part of the discussion in this section is related to the risk-acceptance question.

\section{A. The Multiphase Character of Developments}

The development of major new technologies, or processes or methods in nontechnological areas, generally proceeds through several distinctly different phases. This fact is very well known, but its application to the discussion of safety seems to be lacking.

1. Exploratory and Experimental Phase. The information derived during this phase shows the basic economic prospects as well as the possible risk of the new method. If this information is satisfactory, the next phase can be started.

2. Developmental Phase. During this phase, a first full-scale version of the new method is actually being developed. Along with the development, more detailed information on risk is obtained. Most of this information on risk could not have been obtained during Phase 1 since many specifications were not 
yet defined or developed at this time. If the developmental phase is successful and the residual risk can be made small enough, the method can be practically applied.

3. Introductory Phase. The first applications of the new method or technology may be identified as an introductory phase. The experiences during this phase, which in a sense represents a life test on a sizable scale, lead to refinements of the first developed version.

4. Application of a Mature Technology. Phase 3 gradually develops into Phase 4, when further changes on the method become smaller and smaller.

The recognition of the multiphase character of major technological developments to the development of fast breeder reactors has several implications for the discussion of safety and risk.

1. Fast breeder reactors cannot be expected to be developed in Phase 2 with their eventual safety characteristics.

2. The accuracy of the knowledge of the risk of early plants cannot be expected to be as high as it will be eventually. Conservatism in design is the classical method wlaich is applied in early designs to increase the confidence that the residual risk is sufficiently small, in spite of significant inaccuracies.

3. It is consistent with the multiphase development that undue conservatism be eliminated after increased knowledge shows that earlier overdesign is not needed anymore.

4. The multiplication of early risk estimates with large numbers, such as a factor of 1000 for 1000 plants, does not lead to a proper estimate of the risk involved during Phase 4.

5. The logic of the multiphase character of developments suggests that improvements that are developed during Phase 3 are implemented in future plants. The opposite approach (backfitting) is directed toward the past and not toward the future. Backfitting, due to its focusing on the past, may actually be detrimental to the development of optimum safety characteristics for Phase 4. It is more likely that the explicit and consequential recognition of evolutionary nature of the safety characteristics of fast breeder reactors leads to safer and more economical plants than any other approach.

\section{B. Long-term Testing of Safety Assessment.}

The probability for major accidents in fast breeder reactors is being made so small that no actuarial accident information can reasonably be expected. Thus, there will never be an actuariai base for testing the riskassessment results. 
Majior accident.s can only result from very unlikely combinations of circumstances following an initiating failure. These initiating failures are much more likely than the progression into a major accident; e.g., complete failure of the diverse alternative scram system is not likely to occur at the same time an independent incident occurs. One must therefore expect that the number of minor incidents per major accidents is extremely large, e.g., that thousands or tens of thousands of minor incidents occur before a significant probability for a major accident is accumulated. This comparatively large number of minor incidents or benign accidents can be used, in the long run, to accumulate an actuarial basis and to provide broad confidence into the specific probabilistic risk assessment. The possibility and the significance of such a test of the risk-assessment results have been pointed out by Avery. ${ }^{18}$

\section{Risks in Perspective}

Any meaningful consideration of the question of the acceptability of a specific risk requires that this risk be viewed in the full perspective of other risks. The full perspective of risk comparison has three different dimensions.

The first dimension of the full perspective is made up of risks of causes, which are unrelated to the risk in question. These risks include two groups, the first of which consists of those imposed by the natural environment, such as lightning, tornadoes, hurricanes, and floods, as well as the impact of cosmic radiation and radioactivity. The second group in the first dimension consists of the risks resulting from other activities, such as transportation and other professional and private activities.

Table I gives a list of accidental risk as it results from acute fatalities. The risks that result from cosmic rays, natural radioactivity, $\mathrm{X}$ - ray exposure, etc., are not included in Table I since they do not lead to acute fatalities. The last line in Table I gives the risk of 100 nuclear reactors (LWR type) for comparison. This latter risk is calculated in Ref. 1. The risk of 100 reactors of the currently installed LWR type is assessed in Ref. 1 to be very much smaller than all other risks listed in Table I. Since there is no reason for the risk of fast breeder reactors (FBR's) to be of a different order of magnitude than the risk of LWR's, the risk of FBR's would also appear at the bottom of Table I.

TAas I. Individual Risk of Acute Fatality by Various Causes itrom p. 230 of Ret. it

\begin{tabular}{|c|c|c|c|c|c|}
\hline Accident type & $\begin{array}{l}\text { Total Number } \\
\text { for toes }\end{array}$ & $\begin{array}{c}\text { Aporoximate } \\
\text { Individual Risk, } \\
\text { Acute Fataiity } \\
\text { Probability/yra }\end{array}$ & Accisent iype & $\begin{array}{l}\text { Total Number } \\
\text { for 1969 }\end{array}$ & $\begin{array}{l}\text { Approximate } \\
\text { Individust Risk, } \\
\text { Acute Fatality } \\
\text { Prebability/yr }\end{array}$ \\
\hline Asotor vehicle & 58.791 & $3 \times 10^{-4}$ & Falling objects & 1.271 & $6 \times 10^{-6}$ \\
\hline Fats & 17,827 & $9 \times 10^{-5}$ & Electrocution & i.148 & $6 \times 10^{-6}$ \\
\hline Fires and hot substance & 7.451 & $4 \times 10^{-5}$ & Railuay & 884 & $4 \times 10^{-6}$ \\
\hline Orswning & 6.181 & $3 \times 10^{-5}$ & tightning & 160 & $5 \times 10^{-7}$ \\
\hline Poiser: & 4.516 & $2 \times 10^{-5}$ & Tornadoes & $9 j^{b}$ & $4 \times 10^{-7}$ \\
\hline Firearnas & 2.309 & $3 \times 10^{-5}$ & Hurricanes & $95^{5}$ & $4 \times 10^{-7}$ ? \\
\hline Machinery :1960 & 2.054 & $i \times 10^{-5}$ & All others & 8.695 & $4 \times 10^{-5}$ \\
\hline Water transpen! & 1.743 & $9 \times 10^{-6}$ & All accidents & & $6 \times 10^{-4}$ \\
\hline Aif Irawi & $1.7 n$ & $9 \times 10^{-6}$ & Nuclear accisents 1000 resctors) & 0 & $3 \times 10^{-94}$ \\
\hline
\end{tabular}

agside on total U. S. population, except as noted.

Bw53-1971 avetage.

(5901-1972 astrage.

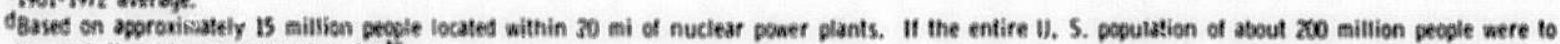
be used, the value would be $2 \times 10^{\circ}$ : 
The first dimension is composed of the risks of unrelated causes or activities. In a second dimension of the full perspective, one considers the risks of directly related activities. This "relation" is mostly investigated in the form of a substitution of activities.

In the simplest form of the investigation of the substitutional risk, a one-to-one substitution is considered; e.g., a risk of a 1000-MWe power plant of one type is compared with a 1000-MWe power plant of another type (e.g., Ref. 15). In the general case, the substitution includes different activities; e.g., a certain increase in the power-generation capacity, including the associated benefit and risk, is compared with a different increase in power generation together with different benefits and risks. Thus, a benefit-risk consideration becomes a central part of the generalized risk-substitution analysis.

The quantification of the benefit-versus-risk consideration is inherently hampered by the fact that benefits derived from the additional energy production are of a different nature than the associated risks. The benefits include jobs as well as an increase and a broadening of the standard of living; the risks include health hazards and some deterioration of the environment.

A question that arises in addition to the quantification of benefits versus risks is the question of how much risk people are willing to accept for certain benefits (even though both are of a different nature). This question is complicated by the fact that the benefits and the associated risks have a different distribution within the populace. Obviously the risk is not concentrated on that part of the populace that are the main beneficiaries through a broadening of the standard of living.

An investigation by $\operatorname{Sta~}^{16}$ provides some semiquantitative information derived from various activities about a consensus type of a relation of benefits and acceptable risks (see Fig. 6). It is suggested in Starr's study of commonly accepted risks versus benefits that the same semiquantitative relation applies also to nuclear power production.

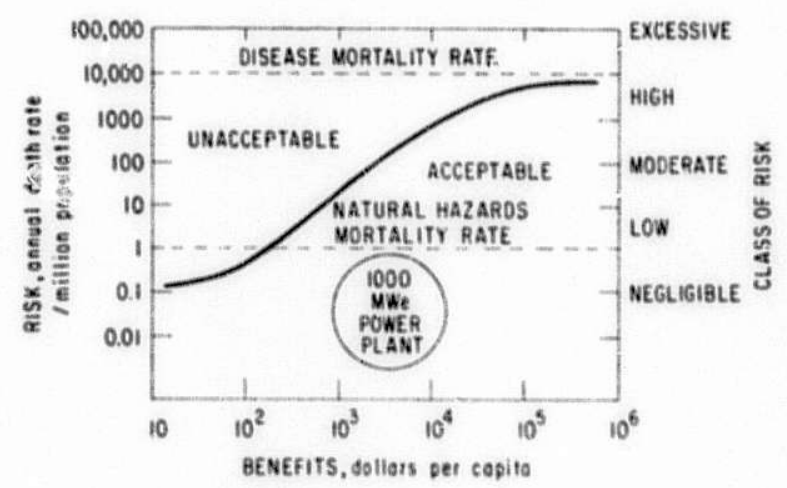

Fig. 6

Benefit-Risk Pattern for Involuntary Exposure (from Ref. 16)

The risks along the first two dimensions of the full perspective can be compared at a given time or be independent of any reference to time (isolated assessments). The risk comparisons in the third dimension take the time dependence explicitly into account. This is particularly of importance for 
major technological developments for which the time between the beginning of the exploratory phase and the time when the matured technology comes in large-scale use amounts to several decades. The current consumption rate of natural resources is so high, and the associated economical, political, and societal changes so substantial, that any meaningful risk assessment of a new technology requires the investigation of this overall impact over a period of several decades. "Isolated" assessme is which are determined without explicit consideration of the long-term impact can only provide the input for an overall risk assessment. Valid conclusions cannot be drawn from isolated assessment alone.

The rationale for the development of the fast breeder reactors is based on long-term considerations of energy-resource depletion. The risk assessment, however, is essentially based on isolated considerations. A proper as sessment of the risk associated with a large-scale deployment of fast breeder reactors and a comparison with alternative strategies can only be obtained irom a long-term overall-risk-assessment methodology in which proper account is taken of the basic economical, political, and societal impacts. Important in this context is the decreasing capability of the economy to generate new capital because of the increasing costs of basic raw materials and the increasing expenses for maintaining a clean environment.

The long-term overall risk comparison of two concepts may have quite a different result than the isolated risk comparison. For example, the concept that appears to have the lower risk in an isolated one-to-one comparis on may have a much larger overall risk if the long-term risk assessment of this concept reveals a significant chance for adverse political, economical, or societal reactions, as they may occur due to reduced availability of energy or due to a substantial increase of its price. Such adverse reactions r.ay include a gradual deterioration of the standard of living as well as violent convulsive events. Only a long-term risk assessment can provide the proper basis for a judgment of the acceptability of certain developmental lines. A long-term risk assessment will likely reveal that the mode of energy production, such as the fast breeder reactor, which can assure a nation of the uninterrupted availability of the desired energy at a stable price, has the lowest long-term risk. 


\section{A GENERALIZED RISK-ASSESSMENT METHODOLOGY FOR FAST BREEDER REACTORS}

A. Introduction, Objective, and Goals of the Generalized Approach

The review of the existing risk-assessment methodology, presented earlier in this report, showed that all assessment methods are based on computational consequence predictions for relatively few accident scenarios. The discussion of the possible impact of uncertainties on the accident progression showed that the prediction of the "average" accident consequences, even for a single scenario, may be strongly influenced by uncertainties in parameters and computer models. This suggests the development of an improved methodology. The methodological improvements have been briefly outlined earlier in this report and are further described here.

The generalized methodology proposed in this report has the objective of explicitly including these uncertainties ir the consequence calculation in order to improve the accuracy of consequence prediction and of risk assessment. The method consists, in general terms, of a close simulation of what one can possibly predict about accident progression and about consequences of successive stages of accidents. The possible prediction consists, due to the impact of uncertainties, of a progressively broadening spectrum of individual accident paths, even for a precisely defined accident initiation. Therefore this approach is called the accidext-spectra-progression (ASP) approach.

Although the approach can be formulated quite simply in general terms, its feasibility is not obvious at all and requires substantial developmental work. The required developments are specifically noted along with the more detailed description of the approach (see Sec. B below).

Since the ASP approach is quite general, simpler methods can be deduced from it by proper reduction of the generality of certain features. The general comparison with other methods is discussed in Sec. C.

Specific goals that may be achieved with the ASP approach include replacement of some concepts that are of questionable applicability, such as "worst case," "bounding case," or "risk envelope," by improved concepts. Furthermore, a detailed description of the possible prediction of accident progression may indicate the most important research and development areas, and it may aiso provide guidance for the most economical improvements of the safety of future power-production plants.

B. The Accident-spectra-progression (ASP) Approach

1. Accident-progression Schematics

The feasibility of the ASP approach requires that the individual phases of the accident progression be investigated separately. Therefore, the 
typical phases that may be distinguished in fast-reactor accidents are discussed first. In addition, the accident progression through these phases is described schematically in order to provide a schematic structure for the mathematical description.

The general progression of fast-reactor accidents may be broken down into 12 phases. The accident initiation, the detection of the initiated accident, and the response of the two independent scram systems are combined in the first phase. Virtually all accidents will be detected and safely shut down due to the high reliability of the detection and the two independent scram sys tems. Only with a very small probability will accidents progress beyond this first phase. However, the entire residual risk results from this small fraction of propagating accidents. The 12 basic sccident phases are listed in the time sequence in which they may occur or exert their impact on the progression of the accident. It is, however, not necessary that an accident progress through all the preceding phases; some phases may not occur, others may not apply.

(1) Initiation and initial response (IIR)

(2) Multichannel accident (MCHA)

(3) Transition phase (TPH)

(4) Core-dis ruptive accident (CDA)

(5) Core-accident propagation (CAP)

(6) Vessel response to pressure (VIRP)

(7) Postaccident heat removal (PAHR)

(8) Vessel response to meltthrough (VRM)

(9) Radioactivity removal in containment (R/A-C)

(10) Containment response to pressure or meltthrough (CR)

(11) Radioactivity propagation (R/A-P)

(12) Impact of radioactivity (I-R/A)

The schematic description of the accident progression is based on these 12 accident phases. Such a representation of a typical accident progression is similar to an event tree in the Rasmussen study. The major cifference is that the main emphasis is placed here on the physical phases of the accident progression, whereas the event trees are based on failure or nonfailure of equipment or functions (see Figs. 4 and 7). Furthermore, each branch in the ASP approach consists of a spectrum of accidents. The schematic description of the accident progression through all phases is called an "accident-progression schematic." schematic:

The following symbols are used to simplify the accident-progression

a. The termination of an accident branch in a phase is indicated by short vertical lines, single or double. A double line $(-1)$ indicates accident termination without damage; a single line $(-1)$, damage to the reactor core. 
b. Binary branching (either, or) is indicated in the usual way $(-[)$; the upper branch represents success and the lower one failure of a function or, in general, the more or lsss advantageous branch of an accident progression.

c. In addition to the mutually exclusive binary branching, a branching point is introduced in which a path splits into two fractions; the accident proceeds along both of these branches simultaneously $(-\subset)$ : "simultaneous branching point."

d. Lack of occurrence or applicability of a certain phase is indicated by a dashed line (--.-).

e. If the further progression of an accident branch is of the same type as one that already appears in the schematic, then it may be merged with another one in order to enhance the clarity of the schematic diagram (J).

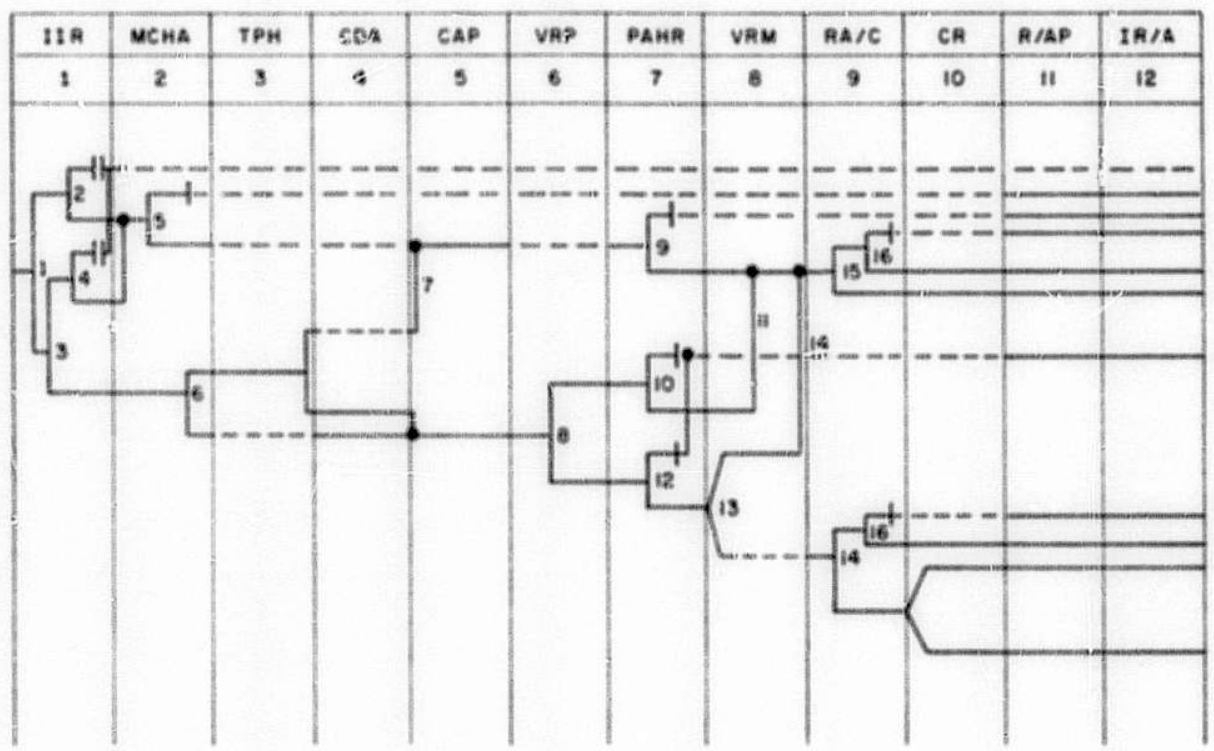

Fig. 7. Iliustration of an Accident-progtession Schematic

Generally, event trees should be drawn on the basis of an actual design. To illustrate the general features involved in an event-tree schematic of a fast-reactor accident, an example of an accident progressi $\mathrm{n}$ schematic is shown in Fig. 7, which is not based on a specific design.

The initial failure may be a coolant flow reduction due to pump soastdown (LOF) or pipe rupture. At branching point 1, the primary scram system responds successfully (upper branch) or it fails (lower branch). At branching point 2 , the progression of the incident is split into two branches: the normal branch of a successful safe shutdown $(-11)$, and a branch for which the flow reduction has been so rapid that the residual fission power and the afterheat leads to sodium boiling. This branch then enters the multichannelaccirlent phase. The resulting multichamel accident may either subside by 
coolant reentry and establishing of cooling through natural convection (upper branch of 5), or the MCHA. may lead to more or less substantial damage of the core. In the latter case the core accident propagates ( $\mathrm{TPH}^{17}$ or CAP), and postaccident heat removal (PAHR) is called for. The PAHR may be successful (upper branch of 9 or not (lower branch). Lack of success of the PAHR automatically leads to melthrough of the vessel. Then, radioactive material enters the containment and must be dealt with. Successful treatment of the radioactivity (upper branch of 14) may either arrest the radioactivity or require some release through a filter (upper and lower branches of 16). Any released radioactivity will propagate $(\mathrm{R} / \mathrm{A}-\mathrm{P})$, and the impact of such an event must be evaluated (I-R/A).

If the primary shutdown system fails (lower branch of 1) the alternative scram system is called for (3). Its successful operation is followed by the branches of 4 , which are, in principle, the same as those following 2.

If the alternative scram system should also fail, an MCHA develops. Should the reactor become permanently subcritica! during the MCHA phase, the further progression of the core accident is principally of the same type as discussed earlier (merged at 7).

If the MCHA does not lead to permanent subcriticality, fuel sweepout or disassembly may be required for the shutdown of the nuclear-accident phase (CDA). Through core-accident propagation, one obtains a pressure loading on the vessel that the vessel can stand or not (8). If yes, the PAHR may provide permanent cooling (upper 10) or not, in which case the vessel will melt through and the further progression may be merged with a path discussed above (merging at 11).

If the vessel is breached by the pressure loading, the PAHR has only minimal chance tis function properly (upper 12); the major branch will describe radioactivity leaving the vessel, either through meltthrough (upper 13) or directly (lower 13; i.e., VRM not applicable). The handling of the radioactivity in the containment will either be successful (upper 14, 15) or not (lower $14,15)$. The residua! release in case of success at 14 or 15 will be filtered or not (upper or lower 16). In case of unsuccessful action at 15 , the containment will be breached either by meltthrough or pressure loating (upper or lower 17).

Probabilities for either branch are associated with the exclusive branching points; "branching ratios" are applied at simultaneous branching points. Both the probabilities and the branching ratios add up to 1 at each branching point.

The probabilities at the branching points in the accident-progression schematic of Fig. 7 are of different types. The failure probabilities of the two scram systems are probabilities for very well-defined events. They may be subjected to a fault-tree analysis. The probabilities at 2 and 4 may have a 
parametric dependency, e.g., for pipe-rupture accidents. The probabilities at 5 or 6 and 8 depend on the severity of the accident progresision, which, in turn, depends on the physics of the entire accident progression as well as on the specific initial burnup condition. A similax situation occurs at other branching points. These probabilities can only be obtained from a computer molel describing the accident progression.

\section{Basic Concepts of the ASP Approach}

The ASP approach uses the following basic concepts in the clescription of the accident progression to incorporate the impact of uncertainties. Some of the concepts listed are cornmonly used in probabilistic accident analysis; others are specific to the ASP approach.

1a) "Accident-initiation categories" are introduced a.s a basis for a complete categorization of possible initiating events. The multitude of different initiating events can be divided into a smail numbar of categrories, sinca there are only a very few basic types of failures involved in accidents (e.g., coolant flow rediction through pipe rupture).

1b) The "occurrence rate" (or frequency) of "initiating failures" in category " $i$ " is denoted by $\mathrm{F}^{i}$, measured in everts of cateogry " $i$ " per yeax. (e.g., frequency for pipe rupture).

1c) The severity of accident initiation within each category generally varies. The variation is discrete, or it may have a parametric depetadence. Let $\mathrm{x}_{\mathrm{i}}$ denote this parameter (or $\mathrm{s}$ et of parameters); then $\mathrm{p}_{\mathrm{i}}(\mathrm{x})$ gives the corresponding probability distribution with*

$$
\int \mathrm{p}_{\mathrm{x}_{\mathrm{i}}}\left(\mathrm{x}_{\mathrm{i}}\right) \mathrm{dx} \mathrm{x}_{\mathrm{i}}=1 \text {. }
$$

Further,

$$
f_{x_{i}}\left(x_{i}\right)=F^{i} p_{x_{i}}\left(x_{i}\right)
$$

describes the frequency distribution of a specific accident out of category $i$.

1d) Additional failures may be encountered during the peogression of an accidient: "encountered failures."

2.) Failures (of operational functions, safety devices, radioactivity barriers, etc.) may be caused by previous damage: "caused failures." Caused failures may occur in a variable degree. Let $\eta$ describe the degree of the failure, with $p_{\eta}(\eta)$ denoting the corresponding probability distributicm. "mhe caused failures generally depend on the degree of the previous damatge, mostly in a continuous fashion. Let $C$ describe the "degree of the damage" at a certain

\footnotetext{
*Integrations of probability distributions are extended over the entire domain, except when a restriction is specifically indicated.
} 
branching point, and let $\mathrm{p}_{\mathrm{C}}(\mathrm{C})$ give the corresponding probability distribution. If the relation of failure and the causing damage is deterministic (for the nondeterministic relation, see point 9), then a unique function $\eta(C)$ describes the failure as it results from a damage C:

$$
\eta(C)=\text { degree of failure } \eta_{1} \text { as caused by a degree of damage } C \text {. }
$$

In most cases, $\eta$ depends monotonically on $C$. The probability distribution for failure, $p_{i}(\eta)$, is then determined by $p_{C}(C)$ and $\eta(C)$ through

$$
p_{\eta}(\eta) d \eta=p_{C}[C(\eta)] \frac{1}{\frac{d \eta}{d C}} d \eta
$$

$C(\eta)$ being the inverse of $\eta(C)$. The damage distribution, $p_{C}(C)$, as well as the failure distribution, $\mathrm{p}_{\eta}(\eta)$, are normalized to 1 . Thus, also the possibilities of zero damage or zero failure are described by the respective distributions, which then contain $\delta$-function components at $C=0$ and $\eta=0$.

3) Generally, "damage" at any stage of the accident progression is a "consequence" of the preceding phase of the accidents. The term "consequence" is usually applied to describe intermediate consequences* as they can be identified at certain stages of the accident progression as well as eventual consequences. Then, $\mathrm{p}_{\mathrm{C}}(\mathrm{C})$ is to be considered as a "consequence" distribution at a certain stage of the accident progression.

4) Figure 8 shows qualitative examples of a consequence distribution and a failure distribution. The $\delta$ functions are drawn as finite-width rectangles in order to indicate the magnitude of the integral over the $\delta$-function component. The failure probability may be obtained by integration. Let $\varepsilon_{\eta}$ denote the upper limit of what may be considered a "practically zero" failure
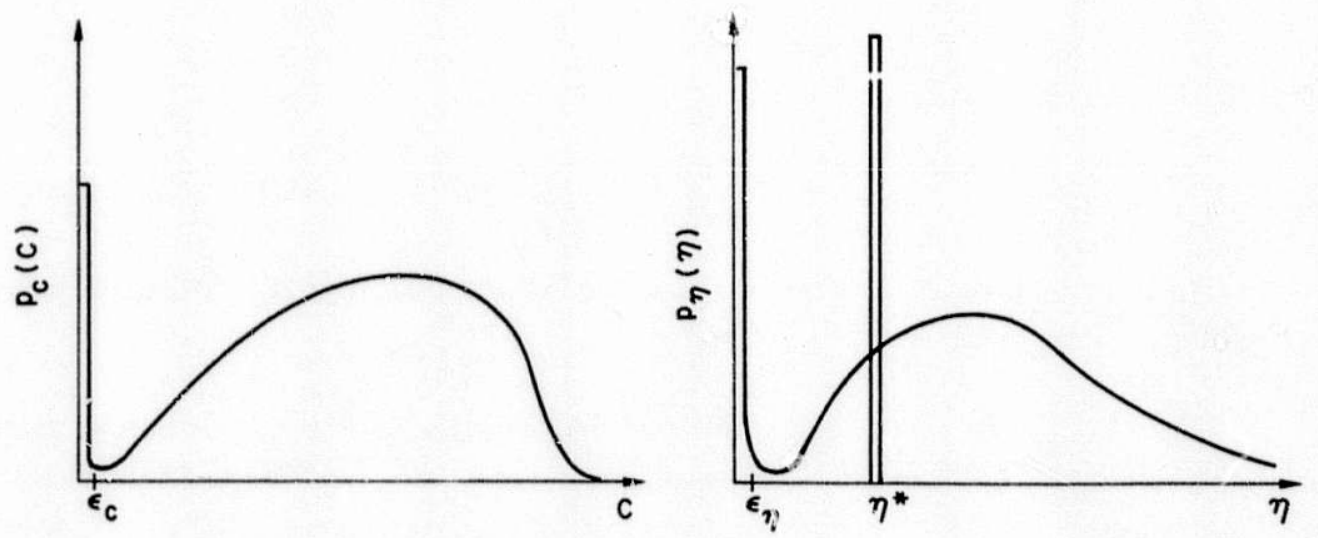

Fig. 8. Illustration of a Consequence Distribution $\mathrm{p}_{C}(C)$ and a Failure Distribution $\mathrm{p}_{\eta}(\eta)$

\footnotetext{
*Examples of intermediate consequences are: fraction of molten fuel, energy release during nuclear transient, and radioactivity release from containment.
} 
(sufficient functioning of the device in question). Then the probabilities for sufficient functioning and for failure are given by

$$
\int_{0}^{€ \eta} \mathrm{p}_{\eta}(\eta) \mathrm{d} \eta=\mathrm{P}_{\eta}=\text { probability for functioning; no failure }
$$

and

$$
\int_{\varepsilon_{\eta}}^{\infty} \mathrm{p}_{\eta}(\eta) \mathrm{d} \eta=\overline{\mathrm{P}}_{\eta}=\begin{aligned}
& \text { probability for caused failure in a specific } \\
& \text { accident progression. }
\end{aligned}
$$

In a simplified treatment, the continuous failure distribution is replaced by two $\delta$-function components. The first $\delta$-function component describes zero failure; the second describes the failure. When the failure is inherently continuous or obviously uncertain, one must, even in the simplified treatment, specify a degree of failure, say $\eta=\eta *$ (e.g., a percentage of fission-gas release). Then the second $\delta$ function is attributed to $\eta=\eta *$.

5) The continuous variation of the distributions of consequences or failures as they have been discussed above result in part from uncertainties of input data and safety parameters, denoted by $a_{j}$. The uncertainty of parameters shall be described by the respective probability distributions:

$$
p_{j}\left(a_{j}\right)=\text { probability distribution for parameter } a_{j} \text {. }
$$

Another contribution to the spread of predicted consequences of degrees of failure is due to uncertainties in special computational models, which, in turn, may result from an uncertainty in our knowledge of the detailed progression of the modeled phenomenon. Uncertainties in computer models of physical phenomena (cladding motion, for example) are not directly amenable to a probabilistic investigation. In the framework of a probabilistic analysis, it is highly desirable to express these uncertainties in parametric form or, if applicable, to replace a set of two different special models by a single unified model comprising both special models. The unified model should contain certain parameters which, in principle, may be used to select either of the special models. A continuous variation of the model parameters allows then a variation of the computational models between or about the two original alternative models.

A special problem arises when two different models for the same phenomenon yield two substantially different results. If one has no experimental indication as to which of the two models is the better, then one may have to split the entire probabilistic analysis at this point into two parts. If one has, however, indications about the likelihood of either model, one may attach probabilities and combine the two parts of the analysis into a single result. It is, however, preferable to further investigate the underlying physics phenomena and to derive a unifying model containing appropriate parameters that may be varied in an uncertainty investigation. 
In summary, uncertainties of computer or physics models should be expressed in parametric form so that they can be incorporated into a probabilistic analysis. This then allows us to express the uncertainty of models by uncertainty distributions of parameters, in this case of model parameters.

6) The "parametric" consequence distribution is denoted by $\mathrm{p}_{\mathrm{C}}\left(\mathrm{C} ; \mathrm{a}_{1}, \ldots, \mathrm{a}_{\mathrm{m}}\right)$. A deterministic calculation of the accident progression along one of the lines of the ASP yields a single value for the consequences. The parametric consequence distribution is then given by the following equation in which the deterministic nature of the consequence prediction is described by a $\delta$ function:

$$
\mathrm{p}_{\mathrm{C}}\left(\mathrm{C} ; \mathrm{a}_{1}, \ldots, \mathrm{a}_{\mathrm{m}}\right)=\delta\left[\mathrm{C}-\mathrm{C}^{\prime}\left(\mathrm{a}_{1}, \ldots, \mathrm{a}_{\mathrm{m}}\right)\right] \mathrm{p}_{\mathrm{a}_{1}}\left(\mathrm{a}_{1}\right) \ldots \mathrm{p}_{\mathrm{a}_{\mathrm{m}}}\left(\mathrm{a}_{\mathrm{m}}\right),
$$

with $C^{\prime}\left(a_{1}, \ldots, a_{m}\right)$ being the single consequence value obtained for the special set of parameters $a_{1}, \ldots, a_{m}$. For simplicity of the nomenclature, let $a_{1}$ represent the initial condition.

$$
a_{1}=x_{i} \text { (characterizes the variable set of initial corditions). }
$$

7) The (complete) consequence distribution is obtained from integrating Eq. 17 with respect to all parameters:

$$
p_{C}(C)=\int \ldots \int p_{C}\left(C ; a_{1}, \ldots, a_{m}\right) d a_{1} \ldots d a_{m} .
$$

Any one of the integrations in Eq. 19 eliminates the $\delta$-function character in the integrand that results from the deterministic treatment. For example, for a single parameter one obtains

$$
\begin{aligned}
\mathrm{p}_{\mathrm{C}}(\mathrm{C}) & =\int \delta\left[C-C^{\prime}(a)\right] \mathrm{p}_{\mathrm{a}}(\mathrm{a}) \mathrm{da}=\int \delta\left(C-\mathbf{C}^{\prime}\right) \mathrm{p}_{\mathrm{a}}\left[\mathrm{a}\left(\mathrm{C}^{\prime}\right)\right] \frac{\mathrm{da}}{\mathrm{dC}^{\prime}} d \mathbf{C}^{\prime} \\
& =\mathrm{p}_{\mathrm{a}}[\mathrm{a}(\mathrm{C})] \frac{\mathrm{da}}{\mathrm{dC}}
\end{aligned}
$$

In all subsequent integrations, the integrand is continuous. In the simple case of a single uncertainty parameter the resulting consequence distribution $20 \mathrm{~b}$ could have been obtained directly, in the same way as Eq. 14 .

8) If the relation of degree of consequences and degree of subsequent caused failures also contains uncertainties, Eq. 13 is to be replaced by $\eta\left(C ; a_{m+1}, \ldots, a_{n}\right)=$ degree of failure $\eta$ as caused by consequence of degree $C$ for parameters $a_{m+1}, \ldots, a_{n}$. 
Then the parametric distribution of caused failures depends on all parameters $a_{1}, \ldots, a_{m}, a_{m+1}, \ldots, a_{n}$ :

$p_{\eta}\left(\eta ; a_{1}, \ldots, a_{n}\right)=p_{C}\left[C\left(\eta ; a_{m+1}, \ldots, a_{n}\right)\right] p_{a_{m+1}}\left(a_{m+1}\right) \ldots p_{a_{n}}\left(a_{n}\right) \frac{d C}{d \eta}$,

which replaces Eq. 14. The failure distribution $\mathrm{p}_{\eta}(\eta)$ is given by an integral of the same type as Eq. 19:

$$
p_{\eta}(\eta)=\int \ldots \int p_{\eta}\left(\eta ; a_{1}, \ldots, a_{n}\right) d a_{1} \ldots d a_{n} .
$$

9) In case of an inherently statistical nature of the relation of the degrees of failure and consequence, the functional relation Eq. 13 is to be replaced by a conditional probability:

$p(\eta \mid C)=$ probability for degree of failure $\eta$ for a given degree of consequence $C$.

The distribution of caused failures is then given by

$$
p_{\eta}(\eta)=\int p(\eta \mid C) p_{C}(C) d C
$$

or, if parametric uncertainties are included, by

$$
p_{\eta}\left(\eta ; a_{1}, \ldots, a_{n}\right)=\int p\left(\eta \mid C ; a_{m+1}, \ldots, a_{n}\right) p_{C}\left(C ; a_{1}, \ldots, a_{n}\right) d C .
$$

10) The deterministic relation 14 is a special case of the more general relation 25. If each value of $C$ leads to a single value of $\eta$, one has

$$
\mathrm{p}(\eta \mid C)=\delta\left[\eta-\eta^{\prime}(C)\right]
$$

and Eq. 25 yields

$$
\left.\begin{array}{rl}
p_{\eta}(\eta) & =\int \delta\left[\eta-\eta^{\prime}(C)\right] p_{C}(C) d C=\int \delta\left(\eta-\eta^{\prime}\right) p_{C}\left[C\left(\eta^{\prime}\right)\right] \frac{d C}{d \eta^{\prime}} d \eta^{\prime} \\
& =p_{C}[C(\eta)] \frac{d C}{d \eta}
\end{array}\right\},
$$

which agrees with Eq. 14 .

\section{Description and Formulation of the ASP Approach}

As indicated above, the ASP approach consists of a close simulation of what one can possibly predict about the accident progression. Part of 
the simulation of the prediction is the explicit treatment of the uncertainties and of their progression through the successive phases.

Phase 1 (initiation and initial response, IIR) begins with the description of the accident initiation and the variety within each of the major accident categories: loss of flow (LOF), pipe rupture, and transient overpower (TOP). The LOF is initiated by loss of power to all pumps. The pump characteristic describes fairly well the resulting flow coastdown. For pipe rupture and TOP, one has a continuous variety of incidents. Either the reactor is safely shut down after such an incident, which is the normal mode of operation, or an accident develops. Then the variety of accident initiators represents the initial conditions for the MCHA phase.

The deterministic treatment of consequences* of the accident progression through this phase for the combinations of varied parameters leads to $\mathrm{C}_{\mathrm{k}}\left(\mathrm{a}_{1}, \ldots, \mathrm{a}_{\mathrm{m}}\right)$, with $\mathrm{k}=2$ for the MCHA phase. A fast code, such as PARSEC, is required to find the consequences for a sufficiently large number of parameter variations. The resulting parametric consequence distribution, which is given by Eq. 17, is then represented by a la rge number of points. It is practically impossible to carry the detailed parametric consecuence distribution into the next phase, and it is clearly not feasible to treat the entire accident progression in terms of parametric consequence distributicns.

A simple numerical example can qualitatively illustrate this point: To represent a parameter distribution in some meaningful way, one needs at least five points: one in the middle and two on either side, because of the nonlinear impact. Suppose there are seven independent parameters appearing in Eq. 17 or 19. The combination of all parameters leads to $5^{7}=10^{5}$ accident paths. Actually, there are more than seven independent parameters; e.g., $2 \times 7=14$ is a more plausible number, which would lead to $10^{10}$ accident histories. Obviously, the number of accident histories must be reduced by many orders of magnitude to make the approach feasible.

A general procedure must therefore be applied in order to reduce drastically the number of accident histories. This procedure consists of the contraction of the information between all accident phases: interphase contraction. The interphase-contraction procedure is an integral part of the ASP approach.

The contraction of information between individual phases of the accident progression is possible without losing significant accuracy (sometimes even none at all), since earlier details may not be neeced for the description of the further progression. This can be seen in the following examples:

a. The consequence of Phase $10(\mathrm{CR})$ is radioactivity release from the containment, with key isotopes specified individually. The detailed sequences that contribute to the release distribution are of no importance for the subsequent impact of this radioactivity.

\footnotetext{
"In general, one has to evaluate several "types" of consequiences.
} 
b. Phase 5 (CAP) leads to a pressure loading on the vessel. Again, the details of the many individual contributors are of no importance for the vessel response.

c. The energy release during a CDA (Phase 4) is primarily determined by the ramp rate of the reactirity insertion and by the "initial" temperature before the final disruptive power burst. How this configuration and its rate of change have been produced in the MCHA phase is of minimal importance.

The effect of the contraction procedure can be illustrated in the numerical example mentioned above. Suppcse contraction is performed between six accident phases by a factor of 25 each. This reduces the original $10^{10}$ his tories by a factor $10^{7}$.

Thus, information contraction between accident phases is very effective in reducing the number of accident paths to be investigated. It makes the entire approach feasible and reduces the information to an amount that may be expected to be a rneaningful result of such a probabilistic analysis.

The interphase information contraction has not only the advantage of making the entire approach feasible, it also forces the investigator to evaluate thoroughly and consistently the complete information at the end of each accident phase. The explicit evaluation of each accident phase leads not only to a much better understanding of the accident progression, it may also provide valuable information for improving the design; it is, furthermore, of extreme importance for the proper assessment of the eventual risk, as discussed in the next section.

The interphase contraction consists of an integration over the parametric variation of $\mathrm{p}_{\mathrm{C}}\left(\mathrm{C} ; \mathrm{a}_{1}, \ldots, \mathrm{a}_{\mathrm{m}}\right)$, e.g., Eq. 19. If a phase contains a large number of uncertainty parameters, it may be necessary to contract the parametric consequence distribution within an accident phase.

retained:

In many cases, some of the parametric variation may have to be

$$
\mathrm{p}_{\mathrm{C}}^{\mathrm{k}}\left(\mathrm{C}_{\mathrm{k}} ; \mathrm{a}_{\mathrm{m}^{\prime}}\right)=\int_{\mathrm{a}_{\mathrm{m} n} \neq \mathrm{a}_{\mathrm{m}^{\prime}}} \ldots \int_{\mathrm{C}^{\mathrm{k}}}^{\mathrm{k}}\left(\mathrm{C}_{\mathrm{k}^{\prime}} ; \mathrm{a}_{1}, \ldots, \mathrm{a}_{\mathrm{m}}\right) \mathrm{da}_{1} \ldots \mathrm{da} \mathrm{a}_{\mathrm{m}}
$$

The average consequence after each phase is given by

$$
\overline{\mathrm{C}}_{\mathrm{k}}=\int \mathrm{C}_{\mathrm{k}} \mathrm{p}_{\mathrm{C}}^{\mathrm{k}}\left(\mathrm{C}_{\mathrm{k}}\right) \mathrm{d} \mathrm{C}_{\mathrm{k}},
$$

with $\mathrm{p}_{\mathrm{C}}^{\mathrm{k}}\left(\mathrm{C}_{\mathrm{k}}\right)$ given by Eq. 19. The parametric consequence distribution $\mathrm{p}_{\mathrm{C}}^{\mathrm{k}}\left(\mathrm{C}_{\mathrm{k}}\right.$; $\left.a_{1}, \ldots, a_{m}\right)$ is normally obtained from a deterministic calculation; it is then equal to a $\delta$ function (see Eq. 17). Thus 
$\bar{C}_{k}=\int C_{k} \int \ldots \int \delta\left[\left(C-C\left(a_{1}, \ldots, a_{m}\right)\right] p_{a_{1}}\left(a_{1}\right) \ldots p_{a_{m}}\left(a_{m}\right) d a_{1} \ldots d a_{m} d C_{k}\right.$

carrying out the $\mathrm{C}_{\mathrm{k}}$ integration gives for $\overline{\mathrm{C}}$ the plausible formula

$$
\overline{\mathrm{C}}_{\mathrm{k}}=\int \ldots \int \mathrm{C}_{\mathrm{k}}\left(\mathrm{a}_{1}, \ldots, \mathrm{a}_{\mathrm{m}}\right) \mathrm{p}_{\mathrm{a}_{1}}\left(\mathrm{a}_{1}\right) \ldots \mathrm{p}_{\mathrm{a}_{\mathrm{m}}}\left(\mathrm{a}_{\mathrm{m}}\right) \mathrm{da}_{1} \ldots \mathrm{da} \mathrm{a}_{\mathrm{m}} .
$$

If the specific consequence calculation involves statistical phenomena, $\mathrm{p}_{\mathrm{C}}^{\mathrm{k}}\left(\mathrm{C}_{\mathrm{k}}\right.$; $\left.a_{1}, \ldots\right)$ is not a $\delta$ function and $\bar{C}$ must be calculated from the general set of equations, Eqs. 29 and 30 .

The distributions $\mathrm{p}_{\mathrm{C}}^{\mathrm{k}}(\mathrm{C})$ obtained from the contraction of the parametric consequence distribution for each accident phase are the basis for the calculation of the "transfer probabilities" $\mathrm{P}_{\mathrm{tr}}^{\mathrm{i}}(\mathrm{k}, \mathrm{b})$, which describe the probability for progression from one phase to the next one:

$P_{t r}^{i}(k, b)=$ probability for "progression" from Phase $k$ - 1 into Phase $k$ along branch $b$ in a category- $i$ accident.

The probability for transfer through the $\mathrm{k}$ Phase along a branch is then given as a product of all the previously encountered transfer probabilities if all probabilities are independent:

$P_{k b}^{i}=\prod_{k^{\prime}=1}^{k} P_{t r}^{i}(k, b)=$ probability for "entering" Phase $k+1$ in branch $b$.

Common-mode failure (e.g., simultaneous failure of the two independent and diverse shutdown systems) required separate considerations which may lead to a substantial increase of the "entering probabilities" $\mathrm{P}_{\mathrm{kb}}$. In most cases, one can combine the dependent events into a single combined event for which the probability is independent of the others (e.g., probability for failure of both scram systems, which is then larger than the product of the individual probabilities). If all dependencies are accounted for by proper combinations, one may then apply Eq. 34 for the combined probabilities.

If the information contraction involves the combination of two or more previously separate branches, then Eq. 34 must be augmented by adding up the different contributions.

The summation over all branches in a given phase, including "nonapplicable" ones (dashed lines in the accident-progression schematic, Fig. 7), must be equal to 1 :

$$
\sum_{b} P_{k b}^{i}=1 .
$$


Incidents are normally terminated in Phase 1, and no radioactivity is released (branches given by

$$
\sum_{b(I I)} P_{k b}^{i}=P_{\| I}^{i}=\text { successful termination probability. }
$$

In all other cases, some sort of an accident occurs:

or

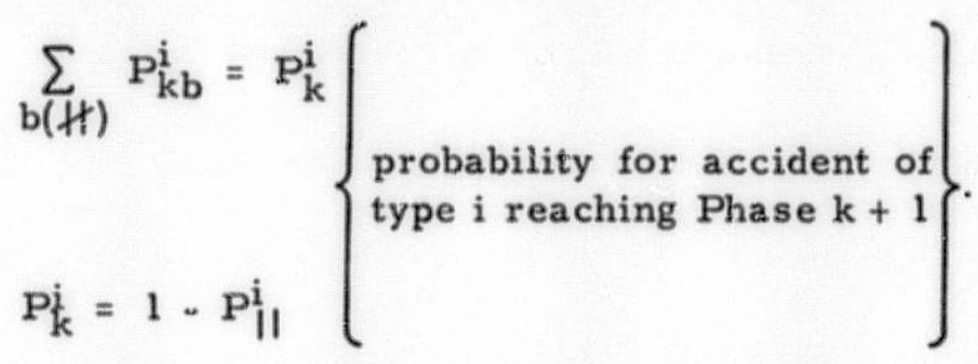

The occurrence rates that correspond to the probabilities defined above are obtained by multiplication with the respective initiation rate $\mathrm{F}^{\mathrm{j}}$; e.g.,

$$
F_{k}^{i}=F^{i} P_{k}^{i}
$$

is the rate of type-i accidents entering Phase $k+1$.

Since the probability for entering Phase $\mathrm{k}$ along branch $\mathrm{b}$ is accounted for separately as described above, the spectrum of accidents entering $\mathrm{k}$ along $\mathrm{b}$ is normalized to 1 .

The consequence distribution after Phase $\mathrm{k}$ is given by Eq. 19. The partial value resulting from branch b may be denoted by $\mathrm{p}_{\mathrm{C}}^{\mathrm{kb}}\left(\mathrm{C}_{\mathrm{k}}\right)$ :

$\mathrm{p}_{\mathrm{C}}^{\mathrm{kb}}\left(\mathrm{C}_{\mathrm{k}}\right)=$ consequence distribution after Phase $\mathrm{k}$ along branch $\mathrm{b}$.

The special value of Eq. 39 for the initiation category i may be denoted by $\mathrm{p}_{\mathrm{C}}^{\mathrm{ikb}}\left(\mathrm{C}_{\mathrm{k}}\right)$.

The occurrence rates of consequences after Phase $\mathrm{k}$ are composed of initial occurrence rates, of the probabilities for entering Phase $k$ along branch $b$, and of the corresponding probability distribution of the consequences, summed over all ibranches:

$$
\sum_{b} F^{i} P_{k b}^{i} p_{C}^{i k b}\left(C_{k}\right)=f_{k}^{i}\left(C_{k}\right) \text {. }
$$

The summation over all initiating events yields the total occurrence-rate distribution of consequences after Phase $\mathrm{k}$ :

$$
f_{k}\left(C_{k}\right)=\sum_{i} f_{k}^{i}\left(C_{k}\right) \text {. }
$$


Of particular importance for safety assessments is the release of radioactivity from the containment (Phase 10). Then, $C_{k}=C_{10}$ describes the radioactivity of various isotopes, in curies, and $f_{10}\left(C_{10}\right)$ gives the occurrence rates of release magnitudes $\mathrm{C}_{10}$.

The complete consequence-occurrence distribution contains a very large contribution at zero consequence, particularly for all incidents that are safely shut down. It may not be very meaningful to include all these incidents into the average occurrence rate and the average consequence. Including or deleting the zero-consequence incidents leads to completely different values.

The total occurrence rate of incidents

$$
F^{\text {all }}=\sum_{\text {all }} F^{i}
$$

may be relatively high if everything is counted. But, since virtually all incidents are terminated with zero consequences, the corresponding average consequence

$$
\bar{C}^{\mathrm{all}}=\frac{1}{F^{\mathrm{all}}} \int \mathrm{Cf} \mathrm{f}^{\mathrm{a} l l}(\mathrm{C}) \mathrm{dC},
$$

is very small.

If, however, the categorization of initiating events is modified in such a way that it includes only incidents having at least a chance to develop into an accident (such as loss of power to the pumps without scram; the case with scram is deleted in the modified definition, since it cannot have any consequence), the corresponding tot $* 1$ occurrence rate, denoted by $F^{\text {acc}}$, is given by

$$
F^{\text {acc }}=\sum_{i(a c c)} F^{i}
$$

which may be very much smaller than $F^{\text {all }}$. The average consequence

$$
\overline{\mathrm{C}}^{\mathrm{acc}}=\frac{1}{\mathrm{~F}^{\mathrm{acc}}} \int \mathrm{Cf^{ \textrm {acc } }}(\mathrm{C}) \mathrm{dC}
$$

will be larger by the ratio $F^{\text {all }} / F^{\text {acc}}$, since the integrals in Eqs. 43 and 45 havi the same value; the deletion of zero-consequence events in the transition from $f^{\text {all }}(C)$ into $f^{a c c}(C)$ does not change the $C$-weighted integral. Therefore, the average risk rate, $\bar{R}=\bar{C} F$, is also not influenced by the deletion of zerocons equence events.

Since Eqs. 44 and 45 are more useful for the assessment of the residual risk, only these equations will be used in the following, and the superscripts "acc" will be deleted: 


$$
\begin{aligned}
f & =F^{a c c} ; \\
\bar{C} & =\bar{C}^{a c c} ; \\
F(C) & =F^{a c c}(C) .
\end{aligned}
$$

The incidents that have been deleted in the transition from Eq. 42 to Eq. 44 will be called "insignificant incidents"; those included in Eq. 44 will be called "significant incidents."

The derivations presented above consider all uncertainties and inaccuracies as they are encountered during accident progression. As a result of these uncertainties, one obtains consequence distributions that may be rather broad, and the resulting average consequence might be very different from the result of most. individual scenarios.

Further uncertainties are introduced by the uncertainty of the occurrence rates $\mathrm{F}^{\mathrm{i}}$ of the initiating events. These uncertainties are explicitly and in detail treated in the Rasmussen report. ${ }^{1}$ A. "confidence band," instead of a single F(C) curve, may represent these uncertainties. Such a band may contain, e.g., $90 \%$ of the initiating events; $5 \%$ are left out on both ends (see Fig. 3).

The elements of the ASP approach for the probabilistic accident analysis as described above consist in summary of the following steps.

a. Treatment of the accident progression in each phase, for the variety of initial conditions and for a proper variation of all parameters. The treatment of each individual accident phase includes, therefore, a full sensitivity study.

b. Superposition of all the resuits of the sensitivity study with their proper weights as obtained from the probability distributions of the pa rameters involved in this phase. The "results" may be considered "consequences" of the accident progression through this phase.* The properly weighted parametric consequence distribution is given by Eq. 17.

c. Interphase information contraction, i.e., a representation of a large number of points of the parametric consequence distribution, calculated in step b by a much smaller number of points (or histogram values). The interphase contraction consists of an integration of the parametric cons equence distribution over most or all of the uncertainty parameters; see Eq. 19 or 29.

\footnotetext{
"The accident progression through some of the phases leads to caused failures. The probability distribution for the degree of failure may be obtained by utilizing the concepts described above (see Sec. IV.B.2 above).
} 
calculates:

d. From the variety of consequences, obtained in this way, one

(1) The probabilities for the progression to the subsequent phase in either of the two branches defined in the accident-progression schematics.

(2) The variety of initial conditions in the form of a distrikution that is normalized to 1 .

e. Treatment of the subsequent phase as in steps a-d.

4. Discussion of Significance and Feasibility of the ASP Approach

The ASP approach can be characterized as a large-scale sensitivity analysis in which the parameter variations are constrained by the appropriate probability distributions. Sensitivity analysis is quite common in safety evaluations. The new feature added here is the consistent constraint by the probability distributions of all parameters. Furthermore, this constrained sensitivity analysis is consistently carried through the entire accident progression.

The four major advantages of the ASP approach are:

a. It properly includes the uncertainties in the calculation of the average risk and thus provides a more accurate value for the most important quantity of assessing the risik to the public.

b. It provides the proper assessment of the variation of the risk as it results from uncertainties in parameters and models. Thus, s sequence of unlikely assumptions as they are often made in bounding or worst-case calculations will :ead to accident paths which, in the ASP analysis, has only a minuscule contribution to the average residual risk. Putting bounding and worst-case risk estimates in the proper perspective as provided by a consistent description should help to alleviate worthless and distractive investigations and discussions.

c. A detailed probabilistic analysis oi the entire ac cient progression provides a consistent way to derive an optimum set of design implica tions such that the highest degree of safety can be obtained at reascthble cost, or an acceptable degree of safety can be achieved with minimal cost.

d. A detailed probabilistic analysis also helps to optimice the allocation of further near- and long-term resesch and developinent expditures.

The feasibility of the ASP approach hirges on three substantial requirements which are not yet satisfied at present. These three requiremints are:

a. Availability of the parameter distributions, $\mathrm{p}_{\mathrm{a}}(\mathrm{a})$.

b. Expression of uncertainties of models in parametric form.

c. Very fast computational models for the various accident ph.tses.

These three requirement:s will be discussed in the following. 
Probability distributions of most parameters are not available.

However, for all directly appearing (and measurable) parameters, such as heat conductivity, Doppler coefficient, and sodium-void reactivity, one has from experiments or from different calculations a good indication of their uncertainty range. The uncertainty distributions of some parameters may be sir,ilar to a Gaussian distribution; for others, one may have no knowledge at all about the shape of the distribution. Then a "range" may be considered as the simplest distribution, i.e., a constant within a chosen range and zero outside of it. However, such a basic "block" distribution needs to be modified for two reasons:

a. The chosen uncertainty range is uncertain in itself; i.e., instead of a single block distribution, one should rather consider a superposition of a variety of block distributions.

b. Parameter values have a lower probability with increasing distance from the average vriuc, even for a given width (or range).

Both reasons require the distribution to be tapered off on both ends. One may further want to avoid unreasonable values, wich as a negative heat capacity or a positive Doppler coefficient, by restricting the distribution to a reasonable domain (see Fig. 9).

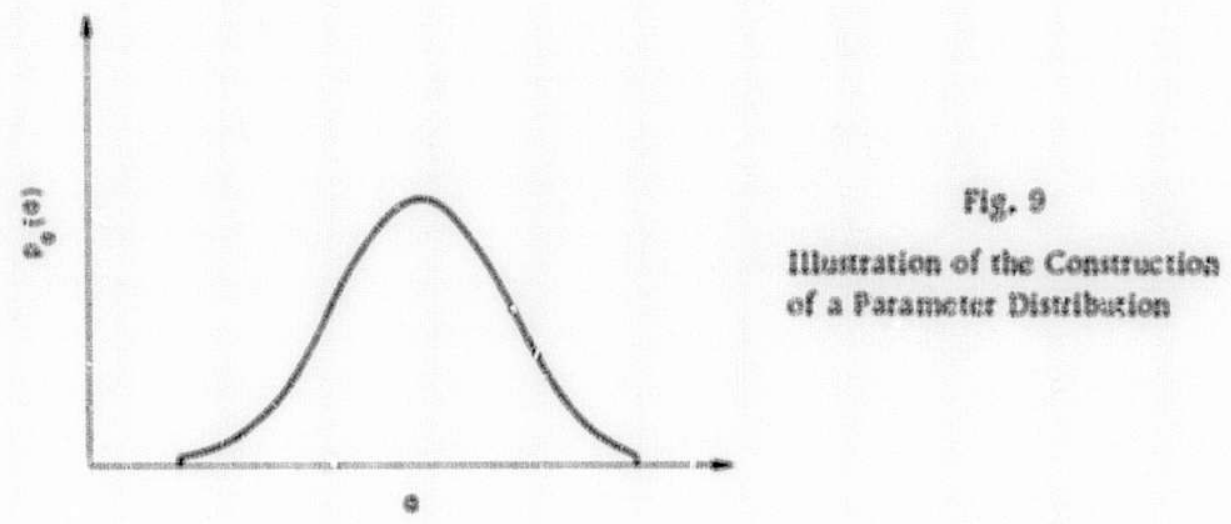

The construction of parameter distributions as described above is plausibis, but in its details arbitrary, since in most cases there is not enough information to thenretically derive the shape of the useertainty distribution. One would expect, however, that the details of the probability distribution are normally of lesser importance than the fact that the parameter is dis * tributed at all about a reasonabie range.

11, however, in an exceptional case, the resilts shotild be very sensitive to the magnitude of a parameter variation in either direction; one has identified an important item for further investigation. Special experinental and theoretical investigations would then have to be performed to explore, in more detail, the parameter variation on the sansitive wing of the originally assumed distribution. 
The uncertainties of physics models and corresponding computational models are currently not expressed in parametric form. A specific effort is required to express model uncertainties in parametric form.

The development of very fast computational models for the MCHA phases has been undertaken in an ANL-Purdue cooperative effort. The development of similar models for the other accident phases is required for a complete probabilistic description of the accident progression.

In general, the accuracies of very fast computational models must be expected to be lower* than those of the detailed codes (such as the SAS code). However, this does not hamper the applicability of the fast models for probabilistic investigations. These models are applied to calculate the variety of consequences as they result from the variation of all parameters. The inaccuracy of the fast model only need to substantially less inan the variation to be investigated. Since the variations to be investigated are probably large, it should be feasible to develop fast computational models of sufficient accuracy.

\section{General Comparison with Other Methods}

Since no results are available from the proposed approach, a comparison with other methods is restricted to a qualitative discussion that concentrates on the structure of the results. The comparative discussion is based on the release of radioactivity from tinecontainment (i.e., on the end of Phase 10 in Fig. 7). The further propagation of radioactivity is essentially the same for fast and thermal reactors. Therefore, the same sophisticated treatment that was developed in the Rasmussen Study ${ }^{i}$ can be applied to fast-reactor safety investigations. If the same treatment for propagation of the radioactivity and of its further impact is applied for all assessment methods, the differences in the eventual risk are already reflected in the release of radioactivity from the containment. This justifies the comparison on this level.

\section{Comparison with an Analysig Comprising Deterministic Scenarios}

Computer models for certain phases of the accident progression normally yield a unique result for a given set of initial conditions; i.e., the result deterministically depends on the initial conditions. If such an analysis is carried through the first 10 accident phases (see 5 ig. 7) with only one initial condition for each branch in each phase, one obtains a set of releases after Phase 10, one value for each branch and accident initiator. In a practical application, one augments this approach by a sensitivity analysis of the main parameters and assumptions. The sensitivity analysis is disregarded in this comparison in order to illustrate more clearly several important points.

\footnotetext{
This is not the care fot all aspects of the calculation. A very fast code allows the treatment of features which can practically tot be weated with a detailed code. For example, PARSEC allows the treatment of all individual subasemblies, whereax $\$ A S S A$ lumps the (c.g., 200) subassemblies into 10 channels.
} 


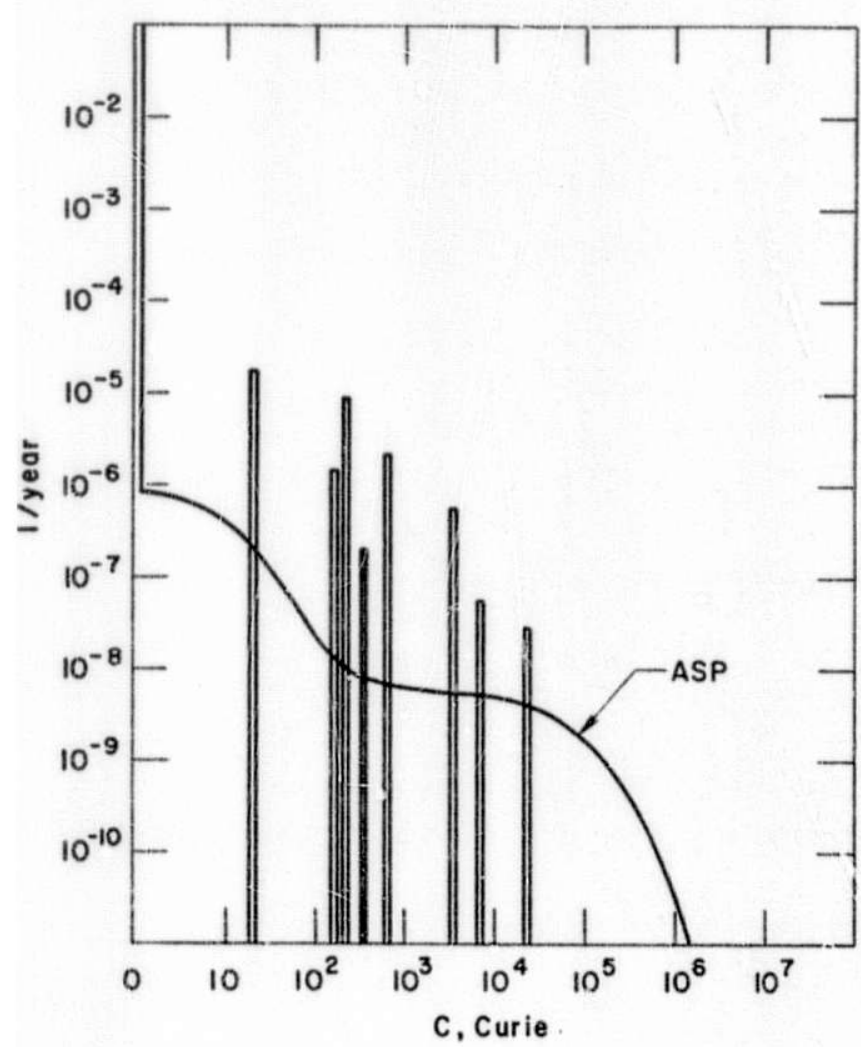

Fig. 10. Qualitative Comparison of (Assumed) Results of the ASP (Curve) and of the Deterministic Scenario (Bars) Approaches

Figure 10 shows the as sumed result of an ASP analysis. It is composed of the contributions of a.ll initiating categories. Let: $\mathrm{C}\left(=\mathrm{C}_{l, 0}\right)$ measure the radioactivity release, in curies. The total release-rate distribution $f(C)$, in release per year and curies, is given by Eq. 41 (with $\mathrm{k}=10$ deleted to simplify the notation):

$$
f(C)=\sum_{i} f^{i}(C)=\sum_{i} F^{i} p_{C}^{i}(C) .
$$

Dividing $\mathrm{f}(\mathrm{C})$ by

$$
F=\sum_{i} F^{i}
$$

gives the total consequence distribution

$$
p_{C}(c)=\frac{1}{F} f(c) .
$$

If the consequence calculation is assumed to be deterministic, one has instead of the broad functions $\mathrm{p}_{\mathrm{C}}^{\mathrm{i}}(\mathrm{C})$ in Eq. 47 a set of $\delta$ functions at the consequence values $C^{i}$ as they are obtained from the deterministic treatment:

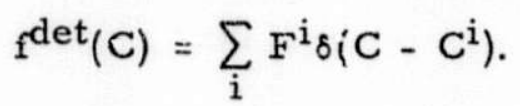

Instead of $\delta$ functions, narrow bars with height proportional to $F^{i}$ are shown in Fig. 10 to illustrate the different factors $F^{i}$.

Since all $p_{C}^{i}(C)$ are normalized, the integrals over Eqs. 47 and 50 have the same value: the total occurrence rate of "significant incidents." Although Eqs. 47 and 50 have the same total integral, any partial integrals will be different, e.g.,

$$
\int_{0}^{\varepsilon} \mathrm{C} \mathrm{p}_{\mathrm{C}}(\mathrm{C}) \mathrm{dC}=\mathrm{P}_{0 \mathrm{C}} \text {, }
$$

which gives the probaisility for practically zexo consequence, and 


$$
\int_{{ }^{e} \mathrm{C}}^{\infty} \mathrm{P}_{\mathrm{C}}(\mathrm{C}) \mathrm{dC}=\mathrm{P}_{\mathrm{C}},
$$

which gives the probability for a significant release, will be different in both approaches.

$$
\begin{aligned}
& \text { Also different are the average release } \\
& \overline{\mathrm{C}}=\int \mathrm{Cp}_{\mathrm{C}}(\mathrm{C}) \mathrm{dC}
\end{aligned}
$$

and the corresponding average risk rate

$$
\bar{R}=F \bar{C}=\int C f(C) d C .
$$

The reason for these differences is that the average values as calculated from the ASP approach will normally be different from the $\mathrm{C}^{i}$ values obtained from the deterministic treatment; i.e.,

$$
\overline{\mathrm{C}}^{\mathrm{i}}=\int \mathrm{Cp}_{\mathrm{C}}^{\mathrm{i}}(\mathrm{C}) \mathrm{dC} \neq \mathrm{C}^{\mathrm{i}} \text {. }
$$

2. Comparison with Farmer's Limit-line Approach

Farmer's approach is conceptually different from the approach presented here. Farmer's limit line is a release criterion and not a consequence distribution. The release criterion limits the domain in which $(F, C)$ points are allowed in the frequency-versus-consequence plane.

To compare the results of the ASP approach with the Farmer curve, one must convert the continuous ASP results into points in the F-C plane. The average consequence $\bar{C}^{i}$ in a sategory-i accident

$$
\overline{\mathrm{C}}^{\mathrm{i}}=\int \mathrm{C}_{\mathrm{P}}^{\mathrm{i}}(\mathrm{C}) \mathrm{dC}
$$

is obviousiy the appropriate abscissa and the corresponding $F^{i}$ the ordinate. The $\left(F^{i}, \bar{C}^{i}\right)$ value can be plotted in the F-C plane (see Fig. 11).

The $f(C)$ curve of the ASP results is also piotted in Fig. 11. Its magnitude is not directly cornparable with Farmer's limit line; the integral of $f(C)$ is equal to $F$, whereas integrating Farmer's release criterion has no direct meaning (see Sec. II. B.6).

The magnitude of the $f(C)$ curve is drawn much lower than the Farmer curve in Fig. 11 so that the average risk as obtained by integration can be equal to the sum of the few points in Fig. 11:

$$
\bar{R}=\sum_{i} F^{i} \bar{C}^{i}=\int C f(C) d C .
$$




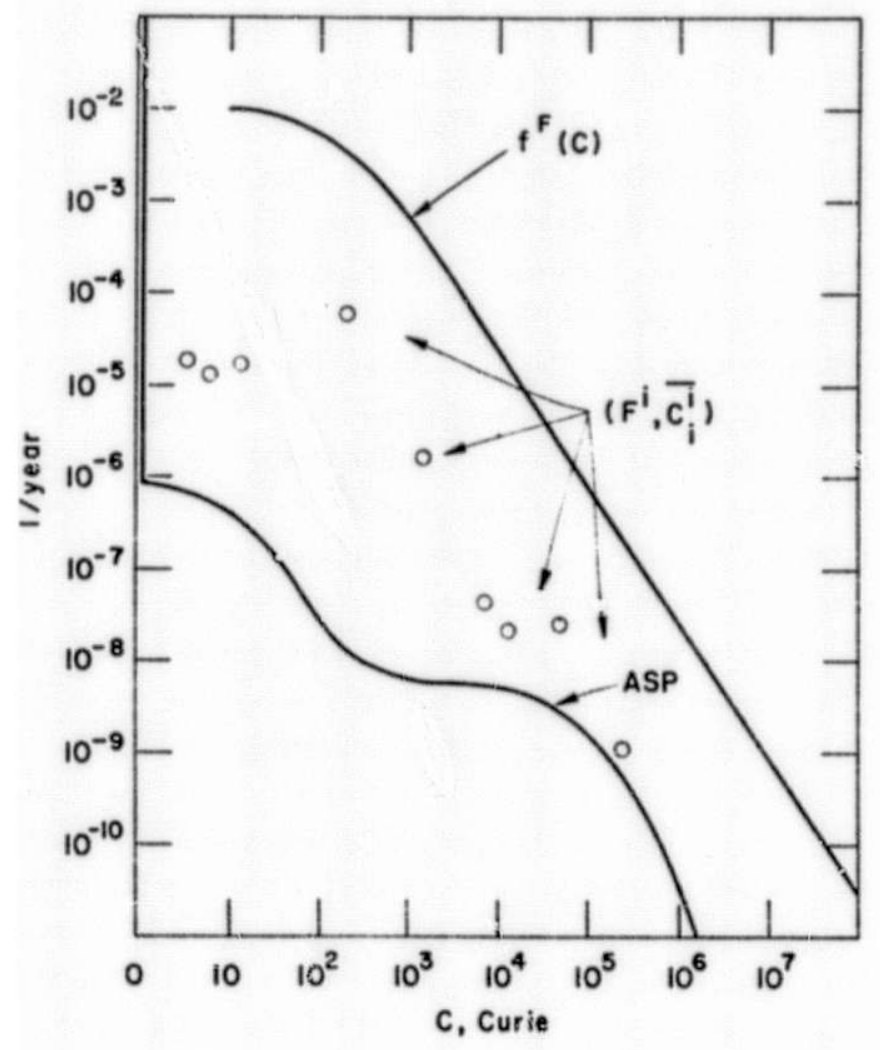

Fig. 11. Comparisolz of (Assumed) Results of the ASP (Lowe: line) and Farmer's Limitline (Upper line) Approaches
Farmer's release criterion has direct implications for the design. If a specific accident scenario yields a point beyond the criterion, the design has to be modified somewhere along the line from accident initiation to the release, such that the modified risk is reduced to a point within the allowed domain. Although Farmer's release criterion provides no direct limitation of the total average risk rate, $\bar{R}$, its magnitude was chosen such that the total risk from the typical set of hypothesized accidents appeared acceptable.

Any approach in which release distributions $\mathrm{f}(\mathrm{C})$ are just calculated, such as in the ASP approach or also in the Rasmussen Study, does not at first provide explicit limitations of the risk of specific scenarios nor of the average risk rate $\bar{R}$. Hovever, an explicit limitation of the average risk

rate can be readily imposed. Also, criteria for risk limitation from individual branches can be introduced: If a particular branch yields an unproportionately high risk as compared to the other branches, the reduction of the risk of that branch may have a significant effect on the overall risk. Design and safety engineers are expected to pay particular attention to unproportionately highrisk contributions.

In addition to the limit that may be imposed on $\bar{R}$, a further criterion for the acceptable risk may be obtained from a proper risk-aversion formula. In general, all these criteria can be imposed, independent of the approach which is applied for the risk calculation as such.

\section{Comparison with Envelope Integration}

A deterministic treatment of several typical accident scenarios yields results that may be plotted on an F-C plane. In the envelope-integ ration approach, one draws an envelope above this set of points and integrates the resulting curve. To have a direct comparison of the ASP and the envelopeinteg ration approaches, the $\left(\mathrm{F}^{\mathrm{i}}, \overline{\mathrm{C}}^{\mathrm{i}}\right)$ points of $\mathrm{Fig} .11$ are reproduced in Fig. 12 and an envelope is drawn around these points. 


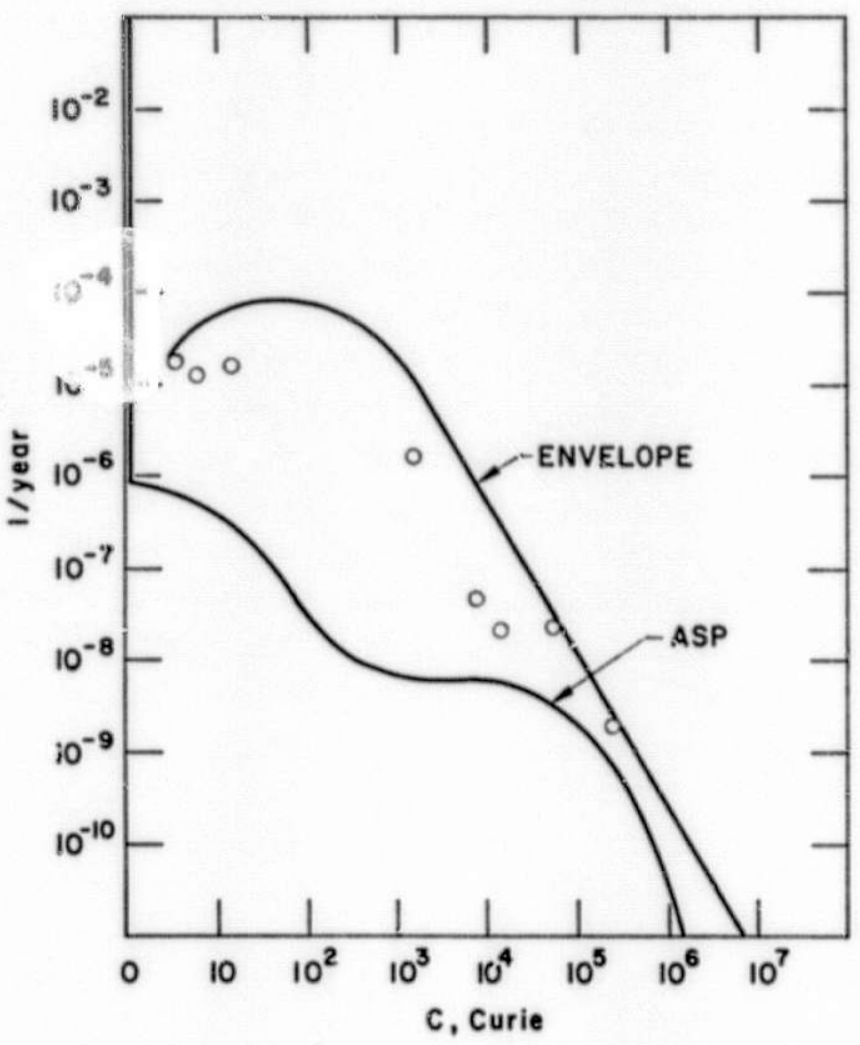

Fig. 12. Comparison of (Assumed) Results of the ASP (Lower line) and the Envelopeintegration Approaches (Upper line)
As discussed above, the $\left(\mathrm{F}^{\mathrm{i}}, \overline{\mathrm{C}}^{\mathrm{i}}\right)$ points a re located well above the $\mathrm{f}(\mathrm{C})$ curve in Fig. 11 since (and if) the entire integral of the $f(C)$ curve is concentrated in a few points. Thus, an envelope around the points in Fig. 12 will obviously be located well above the actual $\mathrm{f}(\mathrm{C})$ curve. The average risk as obtained from integ rating the envelope (times C) may be very different from the one from the ASP analysis. In the example depicted in Fig. 12, the risk from the ASP analysis is much lower than from the envelope integration.

In Sec. II. B. 6, the envelopeinteg ration method was discussed from the general point of view and was found conceptually inappropriate. This view is illustrated by Fig. 12. The $\left(F^{i}, \bar{C}^{i}\right)$ points in Fig. 12 are the integrals and the first moments of the components of the $f(C)$ curve. Thus, the

"correct" continuous distribution that correspords to these points is $\mathrm{f}(\mathrm{C})$. However, the enveloping approach may create a completely different curve, such as the one depicted in Fig. 12.

\section{Comparison with the Rssmussen Approach}

Probability distributions were used in the Rasmussen ${ }^{1}$ Study in great detail for the propagation ${ }^{14}$ of the radioactivity and its impact on the populace. For example, the distribution of weather conditions includes 25 different weathe 1 types, with the information based on the measurements on 40 actual reactor sites. The population distribution was represented in $16 \mathrm{sec}-$ tors as a function of the distances from 66 actual reactor sites.

The uncertainty of the accident progression, however, is not treated in a detailed fashion. The radioactivity release is subdivided into several release categories (nine for PWR and six for BWR), and the result is presented as a histogram. If the release categories in Fig. 3 are converted into actual release values (in curies), one obtains an $\mathrm{f}(\mathrm{C})$ histogram, in full conceptual analogy to the $f(C)$ curve of the ASP approach. Instead of $f(C)$ histog rams, the results of the Rasmussen study are generally represented by the integral $F(C)$, Eq. 3. The calculation of the eventual consequences for the detailed weather and population distributions leads to virtually continuous consequence distributions, and their integrals $F(C)$ may be represented by a continuous curve. 
Generally speaking, the difference between the Rasmussen approach and the ASP approach, which is proposed here, is in the treatment of the propagation of uncertainties through all the phases of the accident investigation. The ASP approach is formulated for a fast-reactor accident investigation for which the explicit consideration of the propagation of unceriainties may have a very significant impact on the calculated consequences.

\section{Summary and Conclusions}

The survey of the safety-assessment methodology reveals its his torical development, which may be characterized by a step-by-step reduction of the individual judgmental component in the assessment: The first assess ment approach, which was based on the concept of "maximum credible accident" (MCA), had a strong judgmestal component in the determination of the credibility of each accident type. The most recent assessment ${ }^{1}$ uses an approach (Rasmussen approach) in which a consistent mathematical structure is applied for the assessment. The application of individual judgment is largely reduced to making a choice for those input quantities that have significant uncertainties. The impact of the uncertainties of failure probabilities are expressed in terms of a confidence band about the average risk.

The methods reviewed are divided into "consequence- and riskoriented approaches." The first category includes the design-basis-accident (DBA) approach, which is currently used in the USA as a basis for reactor licensing. The second group includes all probabilistic approaches that consider the accident probability explicitly together with the consequences. The review concentrated on the probabilistic approaches.

Farmer's "limit-line" approach was historically the first probabilistic approach. It is applied in the UK and some other countries for reactor licensing. It consists of limiting the risk of individual accident scenarios by means of a postulated risk criterion.

Subsequent attempts to find the risk from all types of accidents first by constructing an envelope above the risk values of individual scenarios or by viewing Farmer's limit line as enveiope, and second by integrating the risk under the envelope, have conceptually a poor foundation. Approaches of this kind should be abandoned.

Rasmussen's approach, which has been developed for the reactor safety study, ${ }^{1}$ aims conceptually toward total average risk by assessing sll conceivable contributions (although only the dominating contributions are evaluated numerically). In its methodology and the practically applied detailed computational methods, the Rasmussen study is highly superior to previous reactor safety assessments.

This report describes and proposes a generalized risk-assessment methodology. As in Ref. 1, the accident progression is viewed in its physical sequences and is described by an "accident-progression schematic" that is similar to Rasmussen's event trees. The difference is primarily due to the stronger emphasis on the physical phases of the accident progression rather 
than on failures of functions and barriers. The main feature of the generalized methodology is the consistent description of the uncertainty propagation through the entire accident progression. The uncertainties of input parameters and plysical models are described by appropriate uncertainty distributions. Then, an individual acc *dent path is represented by a propagating spectrum of accidents. Therefore, the approach is called accident-spectra-progression (ASP) approach. The itasibility of the ASP approach requires special developments which are outlined.

In the historical perspective of the developments of risk-assessment methods, the ASP approach can be viewed as a further step in the reduction of the individual judgment. Rather than making a technical judgment on a special choice of input parameters and of computational models, the full variety of choices is investigated. Judgment is applic 'only to the determination of the uncertainty range (and distribution) of those choices.

A special section is deroted to the discussion of the large perspective in which any risk assessment must be viewed as a basis for a judgment of the acceptability of a particular risk. The total perspective is discussed in three dimensions.

In the first dimension, the risk of a given activity (e.g., a 1000-MWe fast breeder reactor) is compared with "unrelated risks," i.e., with risks of activities that have no relation to the risk in question. Acceptability on this level requires that the risk in question be small compared to the already accepted risks of unrelated activities.

The comparisons in the second dimension are concerned with aetivity "substifutions." in a "one-to-cne substitution," one compares the risk of different modes of providing the same power. Also, a benefit-versus-risk consideration has the character of a substitution. One compares the risk of additional power production with obtaining or not obtaining the associated benefits. Acceptability requires the risk of the chosen activity to be smaller than the risk of the alternative choices, including the choice of not obtaining the associated benefits.

The substitutional risk comparisons may yield meaning ful results in a case where the substitution has no major long-term impact. For largescale energy-generating technologies with decades of lead time, substitutions do have a major impact. Then, a long-term overall risk assessment must replace the limited "isolated" comparisons. Part of the long-term overall risk assessment concerns the evaluation of the chances for political, economical, or societal convulsions as they may occur due to reduced availability of energy or due to a substantial increase in its price. Only a long-term risk assessment can provide a basis for a judgment of the acceptability of certain developmental lines. Such a long-term risk assessment will likely reveal that the mode of energy production, such as the fast breeder reactor, that can assure a nation of the uninterrupted availability of energy at a stable price has the lowest longterm risk of all large-scale energy options. 


\section{ACKNOWLEDGMENTS}

This work was made possible by the research appointment of the AUAANL Distinguished Appointment Award, which is gratefully acknowledged. I would like to thank the many members of the Reactor Analysis and Safety Division who contributed through their discussions to this work, particularly, R. Avery, W. R. Bohl, H. K. Fauske, H. H. Hummel, J. F. Jackson, J. F. Marchaterre, L. L. Smith, M. G. Stevenson, and J. B. van Erp. I would also like to thank G. J. Fischer and C. N. Kelber for stimulating discussions on fast-reactor accident analysis, and S. Levine and W. E. Vesely for discussions of the Rasmussen study. 


\section{REFERENCES}

1. Reactor Safety Study, an Assessment of Accident Risks in U. S. Commercial Nuclear Power Plants, WASH-1400 (Draft) (Aug 1974).

2. Theos J. Thompson, private communication (Aug 1968).

3. F. R. Farmer, "Siting Criteria-A New Approach," IAEA Conference on Containment and Siting, Vienna, p. 303 (1967).

4. R. R. Farmer and E. V. Gilby, "A Method of Assessing Fast Keactor Safety," Proceedings of Intermational Conference on the Safety of Fast Reactors, Aix-en-Provence, p. VI-2-1 (1967).

5. G. D. Bell, "Safety Criteria," in Quantitative Safety Analysis, Nuc1. Eng. Design 13, 187 (1970).

6. H. J. Otway and R. C. Erdmann, Reactor Siting and Design from a Risk Viewpoint, Nuc1. Eng. Design 13, 365 (1970).

7. F. R. Farmer, letter to the Editor, Nucl. Safety 13, 362 (1972).

8. R. C. Erdmann, D. Okrent, P. Godbout, and K. A. Solomon, "Fault Tree Analysis of Reactor Safety Systems with Application to the Residual Heat Removal System of a BWR," Proceedings of Calculational Physics Meeting, Ann Arbor, Mich., p. V.47 (1973).

9. N. C. Rasmussen, panel discussion on Risk Assessment of Commerical LightWater Reactors, ANS Meeting, Washington, D.C. (Oct 1974).

10. LMFBR Program Plan, Element 10, Safety, WASH-1110 (2nd Edition), p. 178 (1972).

11. K. E. E1-Sheikh, A New Approach for the Efficient Probabilistic Risk Analysis of Nuclear Power Plants, Trans. Am. Nuc1. Soc. 19, 232 (1974).

12. J. C. Carter, G. J. Fischer, T. J. Heames, D. R. MacFarlane, N. A. McNeal, W. T. Sha, C. K. Sanathanan, and C. K. Youngdah1, SAS1A, A Computer Code for the Analysis of Fast-reactor Power and Flow Transients, ANL-7607 (Oct 1970).

13. F. E. Dunn, G. J. Fischer, T. J. Heames, P. A. Pizzica, N. A. McNeal, W. R. Boh1, and S. M. Prastein, The SAS2A LMFBR Accident-analysis Computer Code, ANL-8138 (Oct 1974).

14. Robert Avery, private communication (1974).

15. C. Starr, M. A. Greenfield, and D. F. Hausknecht, A Comparison of Public Health Risks: Nuclear vs Oil-Fired Power Plants, Nuclear News, p. 37 (Oct 1972). Cf. also UCLA-ENG-7252 (1972).

16. C. Starr, "Benefit-Cost Studies in Socio-Technical Systems," presented at Colloquium for Benefit-Risk Relationships for Decision Making, Washington, D.C. (Apr 1971).

17. J. F. Jackson, M. G. Stevenson, J. F. Marchaterre, R. H. Sevy, R. Avery, and K. 0. Ott, "Trends in LMFBR Hypothetical Accident Analysis," Proceedings of the Fast Feactor Safety Meeting, Beverly Hills, Calif., p. 1241

(Apr 1974). 